\title{
New Iterative Decoding Algorithms for Low-Density Parity-Check (LDPC) Codes
}

by

\author{
Nastaran Mobini, B.Sc. \\ A thesis submitted to the \\ Faculty of Graduate and Postdoctoral Affairs \\ in partial fulfillment of the requirements for the degree of
}

Master of Applied Science in Electrical Engineering

Ottawa-Carleton Institute for Electrical and Computer Engineering

Department of Systems and Computer Engineering

Carleton University

Ottawa, Ontario

August, 2011

(C)Copyright 
Library and Archives

Canada

Published Heritage

Branch

395 Wellington Street

Ottawa ON K1A ON4

Canada
Bibliothèque et

Archives Canada

Direction du

Patrimoine de l'édition

395 , rue Wellington

Ottawa ON K1A ON4

Canada
Your file Votre référence

ISBN: 978-0-494-83044-4

Our file Notre référence

ISBN: 978-0-494-83044-4
NOTICE:

The author has granted a nonexclusive license allowing Library and Archives Canada to reproduce, publish, archive, preserve, conserve, communicate to the public by telecommunication or on the Internet, loan, distribute and sell theses worldwide, for commercial or noncommercial purposes, in microform, paper, electronic and/or any other formats.

The author retains copyright ownership and moral rights in this thesis. Neither the thesis nor substantial extracts from it may be printed or otherwise reproduced without the author's permission.
AVIS:

L'auteur a accordé une licence non exclusive permettant à la Bibliothèque et Archives Canada de reproduire, publier, archiver, sauvegarder, conserver, transmettre au public par télécommunication ou par l'Internet, prêter, distribuer et vendre des thèses partout dans le monde, à des fins commerciales ou autres, sur support microforme, papier, électronique et/ou autres formats.

L'auteur conserve la propriété du droit d'auteur et des droits moraux qui protège cette thèse. $\mathrm{Ni}$ la thèse ni des extraits substantiels de celle-ci ne doivent être imprimés ou autrement reproduits sans son autorisation.
In compliance with the Canadian Privacy Act some supporting forms may have been removed from this thesis.

While these forms may be included in the document page count, their removal does not represent any loss of content from the thesis.
Conformément à la loi canadienne sur la protection de la vie privée, quelques formulaires secondaires ont été enlevés de cette thèse.

Bien que ces formulaires aient inclus dans la pagination, il n'y aura aucun contenu manquant.

\section{Canadä}


The undersigned hereby recommends to the

Faculty of Graduate and Postdoctoral Affairs acceptance of the thesis

\title{
New Iterative Decoding Algorithms for Low-Density Parity-Check (LDPC) Codes
}

\author{
submitted by Nastaran Mobini, B.Sc. \\ in partial fulfillment of the requirements for the degree of \\ Master of Applied Science in Electrical Engineering
}

Professor Claude D'Amours, Examiner

Professor Ian Marsland, Examiner

Professor Amir Banihashemi, Thesis supervisor

Professor Thomas Kunz, Chair 
[iv] 


\section{Abstract}

Low-Density Parity-Check (LDPC) codes have gained lots of popularity due to their capacity achieving/approaching property. This work studies the iterative decoding also known as message-passing algorithms applied to LDPC codes. Belief propagation (BP) algorithm and its approximations, most notably min-sum (MS), are popular iterative decoding algorithms used for LDPC and turbo codes. The thesis is divided in two parts. In the first part, a low-complexity binary message-passing algorithm derived from the dynamic of analog decoders is presented and thoroughly studied. In the second part, a modification for improving the performance of $\mathrm{BP}$ and MS algorithms is proposed. It uses adaptive normalization of variable node messages. 


\section{Table of Contents}

Abstract $\quad$ v

Table of Contents vi

List of Tables $\quad$ viii

List of Figures $\quad$ ix

Nomenclature $\quad$ xi

1 Introduction $\quad 1$

11 Resedreh Motivation and Contributions . . . . . . . . . . . . 1

2 Channel Coding 4

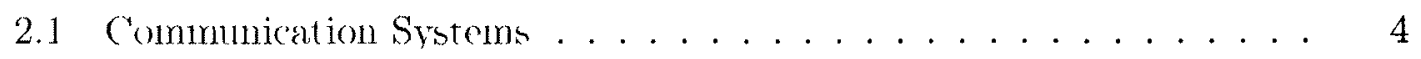

2.2 Error Control Codes . . . . . . . . . . . . . . 5

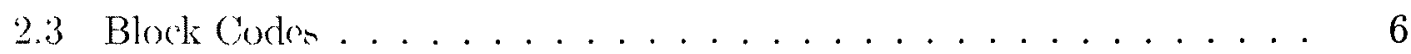

2.4 Low-1)ensity Parity-Check Coles . . . . . . . . . . 8

2.5 Iterative Decoding . . . . . . . . . . . . . . . 10

2.5.1 Hard-docision Algorithms . . . . . . . . . . . . . . 10

2.5.2 Soft-derision Algorithms . . . . . . . . . . . . 11

3 Fixed Point Problem of Iterative Decoding $\quad 14$ 
3.1 Suressive Substitution . . . . . . . . . . . . . . 15

3.2 Solving the First Order Differential Equation . . . . . . . . . . 18

4 Differential Binary Message Passing Decoding 22

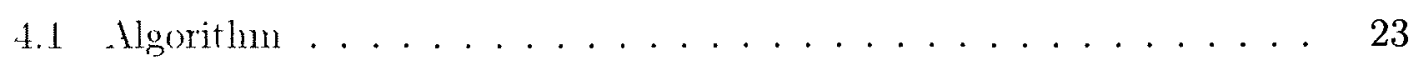

4.1 .1 DD-BMP Algorithm ................... 24

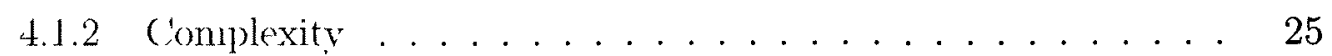

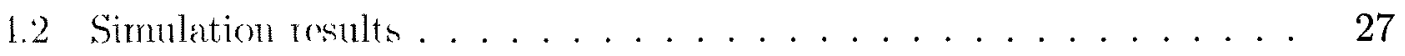

5 Adaptive Belief Propagation Decoding of LDPC Codes 40

J.1 System Morles and the Adaptive Control Tedinirue . . . . . . . . . 42

5.2 Simulation Results and Inalssis . . . . . . . . . . . . 44

6 Conclusions and Future Work 50

List of References $\quad 52$ 


\section{List of Tables}

1.1 Optinal values of $q$ and $\triangle$ for different codes . . . . . . . . 29

5.1 Breakdown of crrors hased on their trpe for the $(1268,456)$ code, decoded by standard BP and adaptice BP, as a function of the maximum number of iterations at different SXR values; " $C$ ". " $P$ " and " $F "$ stand for "Chaotic", "Periodic" and "Fixed-pattern", respectively. . . . . .

5.2 Breakdown of errors based on their type for the $(501,252)$ cokle, decorled by standard BP and adaptive BP. as a function of the maximum number of iterations at different SNR values, "C", "P" and "F" stand for "Chaotic", "I'eriodic" and "Fixed-pattern", mepertively. . . . . 


\section{List of Figures}

2.1 Elements of a communication ststmn. . . . . . . . . . . 4

3.1 Illuatration of the orbit of the function $f(x)=\lambda x(1-x)$ with dilferent valuen of $\lambda$. (a) illustrates an attractive fixed point. (b) repelling fixed point. (c) periodic cucle and (d) chaos. . . . . . . . . . . . . 16

3.2 BER curves of BP. MS. SR-MS for $(1268.456)$ corle. . . . . . . . . . . 19

3.3 Arerage number of iterations vs BER for BP. MS. and SR-MS for $(1268.156)$ code. . . . . . . . . . . . . . . . 20

3.1 BER curves of BP. MS. SR-NS for $(501.252)$ code. . . . . . . . . 21

3.5 Average number of itcration is BER for I3P. MS. and SR-MS for $(504,252)$ corle. . . . . . . . . . . . . . . . . . . . . . .

4.1 BER is. $\triangle$ for different values of $q$ at SNR values $4 \mathrm{~dB}$ and $4.25 \mathrm{~dB}$ for code $(3000,1000)_{(4,6)} \ldots \ldots \ldots \ldots$

1.2 BER curvos of BP, MS. DD-BMP, and ( .1 for codes $(3000.1000)_{(16)}$. $(6000,2000)_{(16)}$ and $(12000.4000)_{(4,6)} \ldots \ldots \ldots \ldots$

1.3 BER curves of BP. MS. DD-BMP, and $G \Lambda$ for code $(1000.2000)$ with different degree distributions. . . . . . . . . . . . 31

4.1 BER (1mves of BP. MS. DD-BMP, and GA for code $(1057,813)_{(3.13)}$.

4.5 BER (urves of BP. MS, IMWBF, DD-BMP, MDD-BMP and GA for code $(273,191)_{(1717)} \ldots \ldots \ldots \ldots \ldots$ 
4.6 BER curves of BP. MS, MMTYBF. DD-BMP. MIDD-BMP and GA for

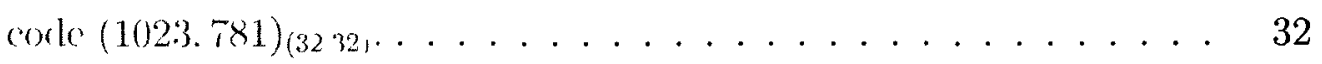

47 BER curres of BP. MS. DD-B.MP, and GA tor irregular code (1008, 504). 33

1.8 Arrage number of iterations vs. BER for BP. MS. DD-BMP and ( $1 A$ for code $(3000,1000)_{(1,6)} \cdot(6000,2000)_{(16)}$ and $(12000.400(0)(16) . \ldots 35$

4.9 Averagr number of iterations vs. BER for BP, MS. DD-BMI and CA for code $(4000.2000)$ with different degree distributions. . . . . . . .

1.10 Average number of iterations rs. BER for BP. MS. DD-BMP, and GA for code $(1057.813)_{(313)} \ldots \ldots \ldots \ldots$

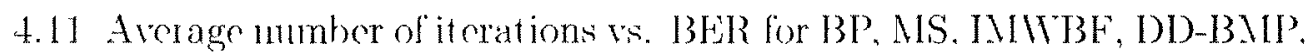
MDD-BNI' and GA for corle $(273.191)_{(1717)} \ldots \ldots \ldots \ldots$

4.12 Average number of iterations vs. BEER for BPP. MS. INIWBF. DD-BAIP. MDD-BMP and (iA tor code $(1023.781)_{(32,32) \ldots \ldots \ldots} \ldots \ldots$. . . .

1.13 Avrage munber of itcrations vs. BER for BP. MS. DD-BMP, and (iA for irregular code $(1008,501) . \ldots \ldots \ldots . \ldots \ldots$

.1 In example of $\mathrm{Eq} .(5.1) \ldots \ldots \ldots \ldots$

5.2 BHR for a) $(1268,456)$ and h) $(504,252)$ LDPC codes decoded by stanlard BP. Normalized BP, and adaptive BP algorithms. . . . . . . . .

5.3 Arerage number of itcration versus $13 E R$ for a) $(1268,456)$ and b) (504.252) LDPC codes decoded br standard BP. Normalized BP. and adaptive BP algorithms. . . . . . . . . . . . . . .

5.4 Iterative decoding trajectories for the (1268.456) LIPC' code at $F_{b} / N_{0}=2.5 \mathrm{~dB}:$ a) number of decision errors versus the iteration uunher. b) $\mathcal{E}(l+1)$ vs. $\mathcal{E}(\iota): 1)$ fixed-pattern 2) periodic-patteru. and 3) random-like or chaotic. . . . . . . . . . . . . . 


\section{Nomenclature}

\section{List of Acronyms}

\begin{tabular}{ll} 
Acronym & Explanation \\
\hline \hline AWGN & Additive White Gaussian Noise \\
BCH & Bose and Ray-Chaudhuri \\
BER & Bit Error Rate \\
BF & Bit Flipping \\
BP & Belief Propagation \\
BPSK & Binary Phase Shift Keying \\
DD-BMP & Differential Decoding - Binary Message Passing \\
IMWBF & Improved Modified Weighted Bit Flipping \\
LDPC & Low-Density Parity-Check \\
LLR & Log-Likelihood Ratio \\
LR & Likelihood Ratio \\
\end{tabular}




\begin{tabular}{ll} 
MB & Majority Based algorithms \\
MWBF & Modified Weighted Bit Flipping \\
WER & Word Error Rate \\
MS & Min-Sum \\
SP & Sum-Product \\
SS & Successive Substitution \\
SR & Successive Relaxation \\
WBF & Weighted Bit Flipping \\
\hline
\end{tabular}




\section{List of Symbols}

\section{Symbol Explanation}
0
all zero-vector or matrix depending on context
1 all-one vector
$\alpha \quad$ normalization parameter
a a node in a Tanner graph
$\mathcal{A} \quad$ coding alphabet
$b \quad$ a node in a Tanner graph
$\beta \quad$ normalization parameter
$B_{a, b}^{\ell} \quad$ memory content of the edge adjacent to node $a$ and $b$ in the $\ell$-th iteration
c
a codeword (a row vector)
$c_{\jmath}$ a check node
$c_{t h} \quad$ clipping threshold
$\mathcal{C}$ a binary linear code
$d_{c} \quad$ degree of check node
$d_{v} \quad$ degree of variable node
$d_{\min } \quad$ minimum distance
$d_{x} / d_{y} \quad$ derivative of $x$ with respect to $y$ 
$E \quad$ number of edges in a Tanner graph

$\mathcal{E} \quad$ a posteriori average entropy

$E_{b} \quad$ energy of an information bit

$f \quad$ variable node operation

$\gamma \quad$ relaxation factor

G generator matrix

H parity-check matrix

$I_{\max } \quad$ maximum number of iterations

$k \quad$ length of message

$\ell \quad$ iteration index

m a message word (a row vector)

$m_{a \rightarrow b}^{\ell} \quad$ a message in LLR domain sent from node $a$ to node $b$ in the $\ell$-th iteration

$n_{i} \quad$ zero-mean Gaussian noise

$N \quad$ block length of code equal to the number of variable nodes

$N_{0} \quad$ one-sided noise power spectral density

$\mathcal{N}(a)$ set of neighboring nodes of the node $a$

$M \quad$ number of check nodes 
$p_{j}^{0}(i) \quad$ probability of $j$-th bit being " 0 " in the $i$-th iteration

$p_{j}^{1}(i)$ probability of $j$-th bit being " 1 " in the $i$-th iteration

$q \quad$ number of quantized bits

Q quantization function

$R \quad$ code rate

$\sigma^{2} \quad$ noise variance

T transpose

$v_{\imath} \quad$ a variable node

x a binary random variable

$y_{\imath} \quad$ channel output corresponding to $c_{\imath}$

$z_{\imath} \quad$ hard decision value corresponding to $i$-th bit 


\section{Chapter 1}

\section{Introduction}

\subsection{Research Motivation and Contributions}

In the current century, the need for efficient and reliable digital data transmission and storage systems has been significantly highlighted. Since 1962, when Gallager introduced low-density parity-check (LDPC) codes along with various iterative decoding algorithms [1], numerous encoding and decoding algorithms have been proposed and standardized to fulfil the need. But it was not until 35 years later that the work of MacKay and Neal $[2,1]$ brought LDPC codes back to light. Because of their very good error correcting performance - close to the Shannon limit - they have been widely studied and became part of various standards in the recent years [1-i].

Iterative decoding algorithms, also known as the message-passing algorithms are used for decoding LDPC codes. An example is the belief propagation (BP) algorithm; theoretically the most complex and the best decoding algorithm from the error rate point of view that has been presented so far. There are lots of published papers with emphasis on reducing the complexity of BP along with preserving its performance. The min-sum (MS) algorithm [ $\xi]$ is a well-known approximation of BP that is much easier to implement. The use of MS algorithm also eliminates the need for estimating the noise power in the communication channel which additionally reduces the 
complexity of the decoder. A review of reduced complexity algorithms can be found in [0]. In contrast with these works, several research groups have aimed to further improve the performance of BP algorithm at the expense of more complexity [1(1-12]. In this thesis we propose one modification to the current algorithms and one new low-complexity high-performance algorithm.

There is always a tradeoff between performance and complexity in the choice of soft-decision and hard-decision algorithms. The hard-decision algorithms in comparison to the soft-decision algorithms are considerably less complex but their performance is not as good as soft-decision algorithms. In the first part of this thesis we propose a new approach in which both the low complexity property of hard-decision algorithms and the good performance property of soft-decision algorithms are preserved. We do this by adding memory with a limited number of bits to the Gallager A algorithm which is a binary message-passing algorithm. The memory improves the error rate of the proposed algorithm with only a moderate increase in complexity.

In second part we will introduce a modified versions of BP and MS algorithm that only slightly changes the original iterative algorithm and can achieve better performance in terms of bit error rate. The first proposed method can be applied to both BP and MS algorithms and originates from the damping idea proposed in [1!]. An adaptive control factor instead of a constant factor for damping is introduced. Although this modification increases the complexity of the iterative decoder, there is a noticeable improvement in bit error rate. The second proposed algorithm concerns the analog implementation of the MS algorithm [1:3]. The Successive Relaxation (SR) model was originally introduced for modeling the dynamics of analog iterative decoders but it also can be used as an LDPC iterative decoder. The idea of using SRMS as an LDPC decoder has been studied in $[11,1.5]$; the promising error correcting property of this algorithm makes it an interesting research topic.

The rest of this thesis is organized as follow. In Chapter 2, a brief review of LDPC 
codes and iterative decoding algorithms is given. Chapter 3 covers the conventional iterative algorithms. A new binary- message passing algorithm is introduced in Chapter 4. Adaptive MS based algorithms is given in Chapter 5. Finally, concluding remarks and future work is addressed in Chapter 6 . 


\section{Chapter 2}

\section{Channel Coding}

\subsection{Communication Systems}

A communication system provides us with a medium for transferring mutual information through free space in the case of wireless communications or a pair of wires, coaxial cable, or optical fibers in wired communications. A typical communication system includes different elements such as the transmitter, transmission channel, and receiver as shown in Fig. 21.

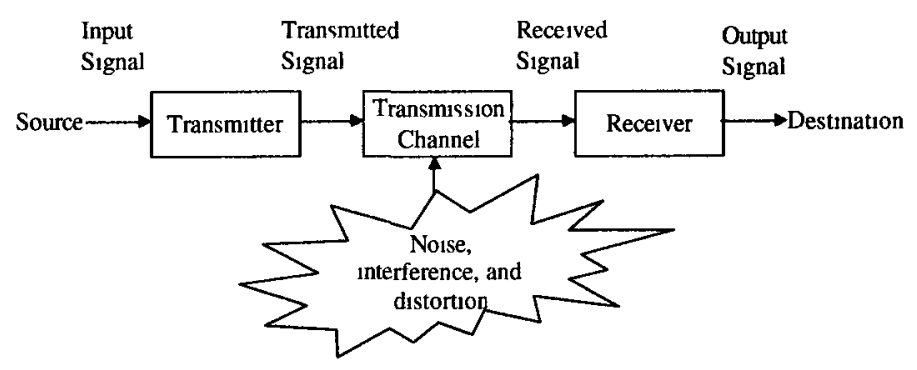

Figure 2.1: Elements of a communication system.

The input signal is processed in the transmitter considering the characteristic of the transmission channel while the process almost always involves modulation and in some cases coding. Then, transmission is conducted via the channel that can cause 
attenuation or loss of signal. The goal of the receiver is to retrieve the input signal that might have changed as a result of distortion, interference, or noise. Demodulation is performed on this received signal. The detected signal usually contains errors which can be minimized through the used of coding. Both modulation and coding are required for reliable long distance transmission. To improve digital communication, different modulation and coding methods are introduced in [ [6, 17].

The input signal may be either continuous or discrete as the case of analog or digital data. Consequently, transmission will be carried on in analog or digital domain, respectively. The emphasis of this thesis is digital communication systems. Hereafter for the sake of simplicity we will assume that the information alphabet is binary i.e. $\mathcal{A} \in\{0,1\}$ and a memoryless binary-input continuous-output channel is used.

For binary data the simplest modulation scheme is binary phase-shift keying (BPSK), the choice of the modulation in this thesis. Before modulation, binary input signals with alphabet of $\mathcal{A}$ are mapped to $\{+1,-1\}$ accordingly. The converted sequence is then mapped into a set of antipodal signals that will be converted to the desired modulation frequency for transmission through additive white Gaussian noise (AWGN) channel, the most commonly used model for communication channels.

\subsection{Error Control Codes}

Claude Shannon created a mathematical foundation for the concept of channel capacity in 1948 [ [ 3 ]. By introducing channel capacity as a limit, he implied that instead of building a very good channel, one can achieve a desirable bit error rate by employing error-control codes. Since then numerous coding schemes have been introduced to approach the Shannon limit. In 1950, the first block codes - a class of singleerror-correcting block codes - with a strong algebraic background were introduced by Hamming [19]. Although Hamming codes provide mild improvement, research on 
the subject continued until the $\mathrm{BCH}$ codes $[20,21]$ and Reed-Solomon codes $[22]$ were created a decade later. Along came the Convolutional codes [:?] and their decoding have been enhanced by the Viterbi algorithm [21]. But it wasn't until the 1990s that turbo codes were able to provide reliable communications with power efficiencies close to the theoretical limit predicted by Shannon. A short time after that, another type of codes known as Low-Density Parity-Check (LDPC) codes [25] with the same capacity approaching property were rediscovered. On additive white Gaussian noise (AWGN) channels, Turbo codes and LDPC codes both closely approach capacity by the use of iterative decoding algorithms at the receiver.

During encoding, based on the input bits, redundant bits - to help the recovery of the lost data - are added to the input sequence in order to form a codeword. To maintain a good performance, the minimum distance between codewords should be maximized. A binary code of size $\mathrm{M}$ has $\mathrm{M}=2^{k}$ binary codewords where $k$ is the number of information bits. The $k$-bits sequences are one by one converted to codewords with block length of $n$, given $n>k$. Such a code is referred as an $(n, k)$ binary code. The amount of redundancy added to the input bits is measured by $1-R$, which $R=k / n$ is called code rate.

\subsection{Block Codes}

A binary linear block code is defined by three parameters: the blocklength $n$, the information length $k$, and the minimum distance $d[26]$. An $(n, k)$ code is able to correct $t$ errors if its minimum distance satisfies the inequality $2 t+1 \leq d$. The larger the minimum distance, the better the code. Finding a good code is an important issue in designing a communication system; the subject has been studied extensively in the literature. In this thesis our focus is not on code design, but on the decoding. 
A subspace of $n$-tuples over $G F(2)$ is a binary linear code $\mathcal{C}$, in which each $n$ tuple that is called a codeword must satisfy the following conditions: the sum of two codewords is another codeword. These codewords are used as the rows of a $k$ by $n$ matrix $\mathbf{G}$ called the generator matrix of the code. The rows of $\mathbf{G}$ are linearly independent, and the number of rows $k$ shows the dimension of the code subspace over $G F(2)$. There are $M=2^{k}$ binary codewords that can be found by

$$
\mathbf{c}=\mathbf{i G}
$$

where $\mathbf{i}$ is a $k$-tuple of information that is encoded to $\mathbf{c}$ the $n$-tuple codeword.

$\mathcal{C}$ as a subspace has an orthogonal complement $\mathcal{C}^{\perp}$ with dimension $n-k$, that is also a subspace and can be used as a code. Therefore $n-k$ linearly independent, $n$-tuples as rows build an $\mathbf{H}$ matrix that is used in finding correct codewords of $\mathcal{C}$. Since a codeword of $\mathcal{C}$ is orthogonal to every row vector of $\mathbf{H}$ then

$$
\mathrm{cH}^{T}=\mathbf{0}
$$

The matrix $\mathbf{H}$ is called the parity-check matrix of the code $\mathcal{C}$. And consequently, the matrix $\mathbf{G}$ is the parity-check matrix of the code $\mathcal{C}^{\perp}$.

Associated with the parity-check matrix there exists a bipartite graph with two sets of nodes, known as a Tanner graph [27]. Considering an $(n, k)$ code, the first set containing $n$ nodes with one by one relation to the codeword bits is called the variable nodes set. The second set, check nodes set, represents the parity bits and consist of $n-k$ check nodes. These two sets are connected according to the parity-check matrix of the code, an edge exists corresponding to each nonzero element in the parity-check matrix. 


\subsection{Low-Density Parity-Check Codes}

Low-density parity-check (LDPC) codes, sometimes called Gallager codes were first proposed by Gallager in his thesis dissertation [25]. At that time they were considered to be very complex due to the computational difficulties of the hardware of that time. It was not until 30 years later that the work of Mackay $[\because, 3]$ brought them back to light. Mackay showed that LDPC code ensembles can closely approach Shannon capacity limit as block length increases [ [] , even closer than turbo codes [ $[3]$. Several important factors such as: the capability of parallel decoding, less complexity per iteration in comparison to that of turbo decoders, natural stopping criterion, error floor at much lower BER than turbo codes, and the flexibility in the choice of rate and codelength, have led them to appear in several standards, such as IEEE 802.16 [i], IEEE 802.11 [6], IEEE 802.3 [1] and DBV-RS2 [7].

LDPC codes belong to the class of linear block codes and therefore are fully described by their parity-check matrix. Their parity-check matrix is very sparse and usually no two rows have more than one column in which they both have nonzero elements (This means no cycle of length four in the Tanner graph). The number of nonzero elements in each column or row is called the degree of that variable or check node, respectively. If all the variable nodes have degree $d_{v}$ and all the check nodes degree $d_{c}$, the code will be a regular LDPC code otherwise an irregular one. The design rate of a regular LDPC code is $R=1-d_{v} / d_{c}$, where all the rows are linearly independent; If not the actual rate will be higher than the design rate. Practical LDPC codes have quite long block lengths while the variable and check node degrees are around 3 or 4 in regular codes or as large as 10 to 15 in irregular codes, therefore the density of nonzero elements in the parity-check matrix is very low - that is why they are called low-density parity-check codes.

Each nonzero element in the parity-check matrix represents an edges in the Tanner 
graph of the code connecting a variable node to a check node or vice versa. Neighboring check nodes of a variable node are labeled by $\mathcal{M}\left(v_{i}\right)=\left\{c_{j}: \mathrm{H}_{j i}=1\right\}$ and the set of variable nodes connected to a check node is denoted by $\mathcal{N}\left(c_{j}\right)=\left\{v_{i}: \mathrm{H}_{j i}=1\right\}$.

Gallager showed that for constructing a good code, the row permutations should be chosen at random out of $n$ ! possible choices. Good LDPC codes that have been found are commonly computer generated and are very long, and because they don't have an algebraic structure their encoding is complicated. Encoding requires the generator matrix which can be found based on $\mathbf{H}$. To enhance the encoding, algebraic LDPC codes based on finite geometries were derived [: well known finite geometries (FG) such as Euclidean and projective geometries over finite fields. In [30], four classes of cyclic or quasi-cyclic FG LDPC codes known as types I and II over these two geometries are described. Both types of FG LDPC codes are regular, and they are the transpose of each other. Their encoding requires simple linear feedback shift registers based on their generator (or characterization) polynomials.

A binary $J \times n$ matrix $\mathbf{H}_{\mathcal{G}}$ whose rows and columns correspond to the lines and points of a finite geometry $\mathcal{G}$ forms a FG LDPC code, if each row has $d_{c}$ and each column $d_{v} 1$ s, while no two rows or columns have more than one 1 in common. $\mathcal{G}$ is a finite geometry with $n$ points and $J$ lines. Each line consists of $d_{c}$ points and two lines are either parallel (no intersection) or share only one point. Every point is the intersect of $d_{v}$ lines and any two points are connected by one and only one line. Extending a finite geometry LDPC codes is possible through splitting each column or row of its parity-check matrix $\mathbf{H}_{\mathcal{G}}$ into multiple columns or rows. If properly done,

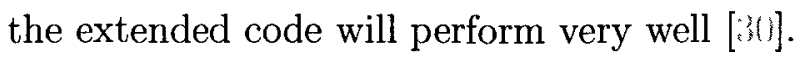




\subsection{Iterative Decoding}

Iterative decoding algorithms based on their alphabet are generally divided into harddecision and soft-decision algorithms. The message alphabet can be as simple as binary, e.g., $\mathcal{A}=\{0,1\}$ or can consist of infinite uncountable number of symbols such as the set of real numbers. If $\mathcal{A}$ is binary, the algorithm is referred to as hard-decision; otherwise, it is called soft-decision. The computational complexity of iterative algorithms usually increases with the alphabet size of messages.

\subsubsection{Hard-decision Algorithms}

In hard-decision algorithms, only binary values are used throughout the decoding process. These algorithms are really important due to their simple implementation. Their binary structure, binary memories and limited wiring, makes them remarkable in hardware implementation especially in the situations where only hard-decision values are available at the receiver. Gallager's algorithm $\mathrm{A}\left(G_{A}\right)[i]$ is, for instance, a hard-decision decoder in the set of Majority Based algorithms $\left(M B^{\omega}\right)$ [31] with alphabet $\mathcal{A}=\{-1,1\}$. In $M B^{\omega}$, a variable node passes its initial channel message to any of its neighboring check nodes unless the majority of its extrinsic incoming messages ( $\left\lceil d_{v} / 2\right\rceil+\omega$ messages) contradict its initial message. In addition, the outgoing message of a check node is the product of its extrinsic incoming messages. The $M B^{\omega}$ algorithms are studied in depth in [3!], by the use of density evolution [:32]. Another example of hard-decision algorithms is Bit Flipping algorithm (BF) [1]. In this algorithm a flipping function is defined that counts the number of unsatisfied syndrome bits (check nodes) in which each variable node participates. Each variable node has a binary buffer to store a hard decision value; the content of this buffer will be flipped if the corresponding output of the flipping function is more than a certain threshold. Decoding continues until all check node equations are satisfied or until the 
maximum number of iterations is reached.

\subsubsection{Soft-decision Algorithms}

In contrast to hard-decision algorithms, soft-decision decoding algorithms such as belief propagation (BP) $[3,2,3,3]$ and Weighted Bit Flipping algorithms (WBF) [ 30$]$ have a continuous alphabet. The BP algorithm is the most complex and the best LDPC decoding algorithm from the error rate point of view. Much work has been done to reduce the complexity of BP with the least effect on its performance. Min-sum (MS) algorithm [`, $111,3,1]$ is a well-known approximation of BP. A review of reduced complexity algorithms can be found in [ 1$]$. The family of WBF algorithms shows a good tradeoff between performance and complexity. Their performance is not as good as that of BP but they are much simpler than BP and its approximations. In other words, they provide good performance/complexity tradeoff. The family consists of WBF algorithm, Modified Weighted Bit Flipping (MWBF) [3i] and Improved Modified Weighted Bit Flipping (IMWBF) [ $\left.{ }^{n}\right]$. The last two schemes are introduced to improve the performance of the original WBF algorithm. Unlike BF, in these algorithms only one bit is switched per iteration. The reliability of the received inputs, which are the absolute values of the detector outputs, are employed in the flipping function and only one hard-decision value associated to the variable node that maximizes the flipping function is flipped.

Starting from WBF, different steps of each algorithm can be summarized in the following lines. The check node process in WBF is finding the minimum reliability among the reliability values of the variable nodes connected to that check node. The flipping function is the weighted sum of the minimum reliability coming from the check nodes connected to a variable node, in which the weighting factor is the syndrome in $\{-1,1\}$ domain. The check node process of MWBF is the same as WBF, but the flipping function is modified by subtracting the weighted reliability value of 
the corresponding variable node from the summation, where the weighting factor is a constant. This modification improves the performance with a slight increase in complexity of the algorithm. In IMWBF, the flipping function is different to some extent. A check node sends different messages to the variable nodes connected to it, in which the reliability of a variable node is excluded while calculating the message going to that node. The flipping function is similar to that of MWBF except it uses the extrinsic incoming messages. This increases the complexity and memory requirement in comparison to the two other algorithms, but the performance is improved.

An iterative algorithm can be implemented in different domains e.g., probability, likelihood ratio (LR) or log-likelihood ratio (LLR). In practice, LLR domain is mostly used since message-passing algorithms are less complex and more robust in this domain. BP algorithm in LLR domain can be summarized as follows. We use the notations $v_{\imath}, i=1, \cdots, N$, and $c_{\boldsymbol{j}}, j=1, \cdots, M$, to denote the set of variable nodes and the set of check nodes, respectively. The message sent from node $a$ to node $b$ is denoted by $m_{a \rightarrow b}$.

\section{Initialization}

Variable node messages are initialized with received values $y_{\imath}$ from AWGN channel $m_{v_{\imath} \rightarrow c_{\jmath}}=\frac{2}{\sigma^{2}} y_{\imath}$ for $i=1, \cdots, N$ and $j=1, \cdots, M$.

2. Check node operation

$$
m_{c_{\jmath} \rightarrow v_{\imath}}=2 \tanh ^{-1}\left(\prod_{v_{k} \in \mathcal{N}\left(c_{\jmath}\right) \backslash v_{\imath}} \tanh \left(\frac{m_{v_{k} \rightarrow c_{\jmath}}}{2}\right)\right)
$$

3. Variable node operation

$$
m_{v_{\imath} \rightarrow c_{\jmath}}=m_{v_{\imath}}+\sum_{c_{k} \in \mathcal{M}\left(v_{\imath}\right) \backslash c_{\jmath}} m_{c_{k} \rightarrow v_{\imath}}
$$


where $m_{v_{\imath}}=\frac{2}{\sigma^{2}} y_{\imath}$.

4. Hard decision

$$
\hat{z}_{v_{\imath}}=m_{v_{\imath}}+\sum_{c_{k} \in \mathcal{M}\left(v_{\imath}\right)} m_{c_{k} \rightarrow v_{\imath}}
$$

Vector $\hat{\mathbf{z}}$ is quantized such that $z_{\imath}=0$ if $\hat{z}_{v_{\imath}} \geq 0$ otherwise $z_{\imath}=1$. If $\mathbf{z} \mathbf{H}^{T}=\mathbf{0}$ decoding is completed, otherwise go to step 2. If a codeword cannot be found within some specified maximum iterations a decoding failure is declared.

Computing the messages in check nodes is computationally complex. As previously mentioned, the most famous algorithm introduced for simplifying BP algorithm is MS algorithm which approximates the check node operation as

$$
m_{c_{\jmath} \rightarrow v_{\imath}}=\left(\prod_{v_{k} \in \mathcal{N}\left(c_{\jmath}\right) \backslash v_{\imath}} \operatorname{sign}\left(m_{v_{k} \rightarrow c_{\jmath}}\right)\right) \times \min _{v_{k} \in \mathcal{N}\left(c_{\jmath}\right) \backslash v_{\imath}}\left|m_{v_{k} \rightarrow c_{\jmath}}\right| .
$$

The operations performed in the variable nodes and for hard decision are those of the BP algorithm. 


\section{Chapter 3}

\section{Fixed Point Problem of Iterative Decoding}

Decoding algorithms are employed to retrieve the original transmitted codeword over a noisy channel. Since the closed-form solution of these algorithms is not obtainable, iterative methods are used to approach an acceptable solution. One important advantage of iterative methods is their adaptivity, the system evolves as new messages are made. Also, they might require fewer computations to get close to the final solution in comparison to the closed-form solution, which is especially attractive for some applications where the need for speed is more critical than the need for precision. Other than convergence speed, convergence itself is an essential issue in real-time systems. For some algorithms convergence can be proved by analytical methods while for others only by computerize simulations. If done experimentally the convergence can be determined for some degree of confidence.

In this chapter, an overview of current iterative methods used for decoding LDPC codes is given. Then the new approach for solving the fixed-point problem of LDPC decoding is presented. 


\subsection{Successive Substitution}

Iterative decoding algorithms aim at solving a non-linear system of $E$ equations with $E$ unknowns, where $E=N \times d_{v}$ is the number of edges in the Tanner graph of a regular LDPC code $[3 ;, 3 \%]$. Solutions to these equations are fixed-points which might lead to a codeword or not. If a codeword is found the decoding is successful otherwise an error or decoding failure is reported. An evolution equation can define the discrete-time dynamical system of iterative decoding algorithm in the following form

$$
\mathbf{x}^{(\ell+1)}=f\left(\mathbf{x}^{(\ell)}\right)
$$

where $f$ is the LDPC decoding function that maps the space $\mathbb{R}^{E}$ into itself and $\mathbf{x}$ is a vector of length $E$ over the space $\mathbb{R}^{E}$ whose initial value $\mathbf{x}^{(0)}$ is arbitrary.

A fixed point $\mathbf{x}$ is found when application of $f$ doesn't change the value, i.e. $\mathbf{x}=f(\mathbf{x})$. Finding fixed-points by putting Eq. (3.1) into the recursion is a classical approach and is called the successive substitution (SS) method [ $; \cdot]$. Most of the conventional iterative decoding algorithms use this approach, e.g. the BP and MS algorithm.

Combining two equations of variable nodes and check nodes of an LDPC decoding algorithm leads to a fixed point problem of

$$
\mathbf{x}^{(\ell+1)}=f\left(\mathbf{x}^{(\ell)}, \mathbf{y}^{(0)}\right)
$$

where $\mathbf{y}^{(0)}$ is the received vector with length $N$. The set of points $\mathbf{x}^{(\ell)}, \ell=0,1,2, \ldots$ is called the orbit of the point $\mathbf{x}$ under the transformation $f$.

Fig. 3.1 shows different behavior of the example function $f(x)=\lambda x(1-x)$ for various $\lambda$ values. We can see (a) attractive fixed point (b) repelling fixed point (c) periodic cycle and $(\mathrm{d})$ chaos. 
(a) $\lambda=2.5$

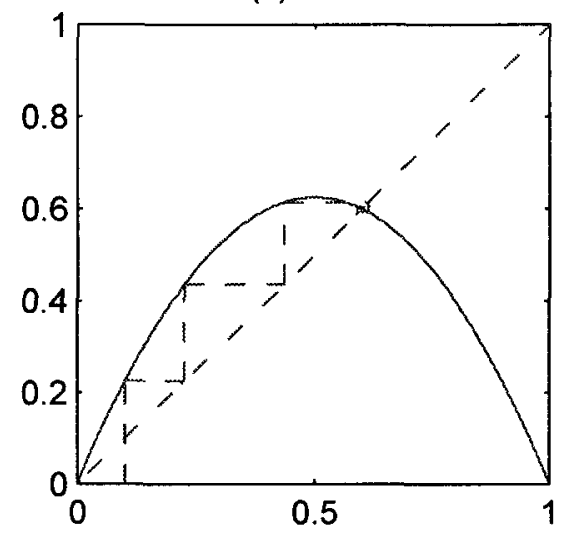

(c) $\lambda=3.2$

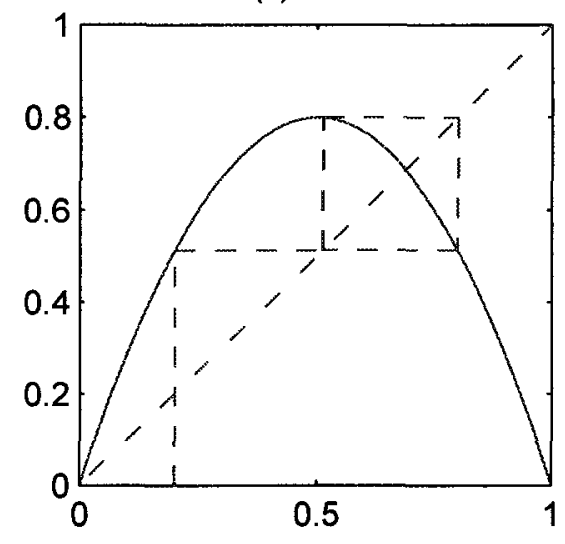

(b) $\lambda=-1$

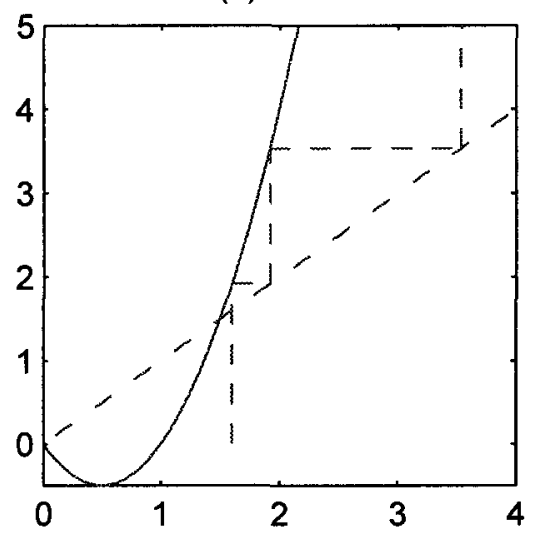

(d) $\lambda=3.9$

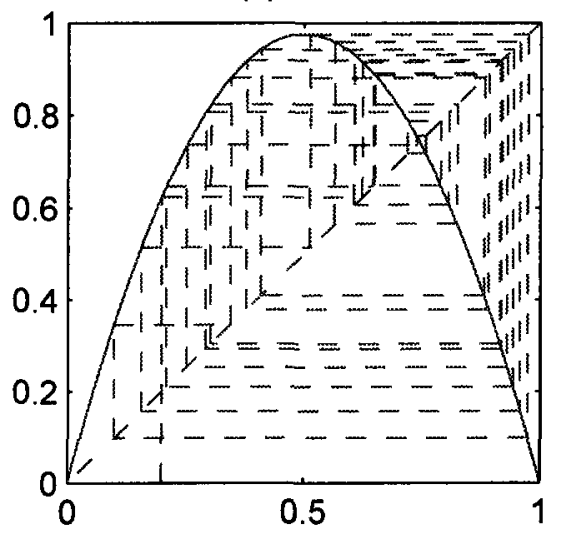

Figure 3.1: Illustration of the orbit of the function $f(x)=\lambda x(1-x)$ with different values of $\lambda$. (a) illustrates an attractive fixed point, (b) repelling fixed point, (c) periodic cycle and (d) chaos. 
In general, iterative algorithms can demonstrate any of the above behaviors known to occur in a nonlinear dynamical systems. Based on the initial vector, the behavior of a general dynamical system lies within following classes:

- Regular attractors: fixed-point, periodic orbits, and limit cycles;

- Irregular attractors: chaotic and strange attractors.

But only one of them, the stable unequivocal fixed-point, is desirable in which estimated LLRs found through iterations are close to negative or positive infinity. When the decoder converges to an indecisive fixed-point that leads to a codeword - estimated LLRs are close to zero - an undetected error is observed which is a serious problem because in real systems this type of error will remain unnoticed and potentially can cause trouble. If the orbit of iterative decoder matches any class other than unequivocal fixed-point usually the chance of observing a codeword approaches zero [3i]. In the case of periodic orbits and limit cycles there is a $k$ in which $\mathbf{x}^{\ell}=\mathbf{x}^{\ell+k}$ and $\mathbf{x}$ never approaches the desired solution similar to chaotic and strange attractors but in the two last ones the behavior of $\mathbf{x}$ is unpredictable. Different methods are introduced to help the convergence of iterative decoders by pulling it out of periodic orbits $[\because],[0]$ or chaos $[3$,$] .$

Numerous numerical methods have been introduced to improve the convergence property of iterative decoders especially turbo decoders [11]. Although for turbo decoders the SS method has the best performance, for LDPC decoders other approaches such as Gauss-Seidel $[\%, 3,1:-11]$ and Successive Relaxation [1:3] have shown promising improvement in performance in comparison to that of SS based approaches. In Section 3.2 these methods are reviewed and a scheme for improving Successive Relaxation method applied to MS algorithm is proposed. But before that we would like to introduce a new modification for conventional implementation of BP algorithm. This modification helps the iterative decoder to converge which means a better error correcting probability. 


\subsection{Solving the First Order Differential Equation}

The first order differential equation

$$
\dot{\mathbf{x}}(t)=f(\mathbf{x}(t))-\mathbf{x}(t), \quad t \geq 0
$$

is interpreted as an analog or continues time representation of the iterative decoder. In $[1,3,1 ;, 10]$ it is pointed out that Eq. (3.3) expresses the dynamic of continuoustime analog iterative decoding.

The under relaxed version of Eq. (3.1) with a relaxation parameter $\gamma \in(0,1]$ can be used to solve the Eq. (3.3). The below method is called Successive Relaxation (SR).

$$
\mathbf{x}^{(\ell+1)}=(1-\gamma) \mathbf{x}^{(\ell)}+\gamma f\left(\mathbf{x}^{(\ell)}\right)
$$

The successive relaxation method employs the current value of $\mathbf{x}$ to estimate the future value. In SS method the value found by $f\left(\mathbf{x}^{(\ell)}\right)$ is considered as the new estimate of the fixed-point in the $\ell+1$ th iteration. But in SR method both $f\left(\mathbf{x}^{(\ell)}\right)$ and $\mathbf{x}^{(\ell)}$ with a certain proportion are used to create the new estimate. For example, SR implementation of MS algorithm is possible by simply modifying the variable node operation and the rest of iterative algorithm is untouched. In MS algorithm $\mathbf{x}$ is the vector of variable node messages $\mathbf{m}_{v \rightarrow c}$ going to check nodes. Updating $\mathbf{m}_{v \rightarrow c}$ is done by doing the original check node and variable node operations represented by $f$ and then involving the current value in the following form

$$
\mathbf{m}_{v \rightarrow c}^{\ell+1}=(1-\lambda) \mathbf{m}_{v \rightarrow c}^{\ell}+\lambda f\left(\mathbf{m}_{v \rightarrow c}^{\ell}\right)
$$

Simulation results have proved the superior performance of SR implementation of MS algorithm over SS implementation [11]. By a slight change in variable node 


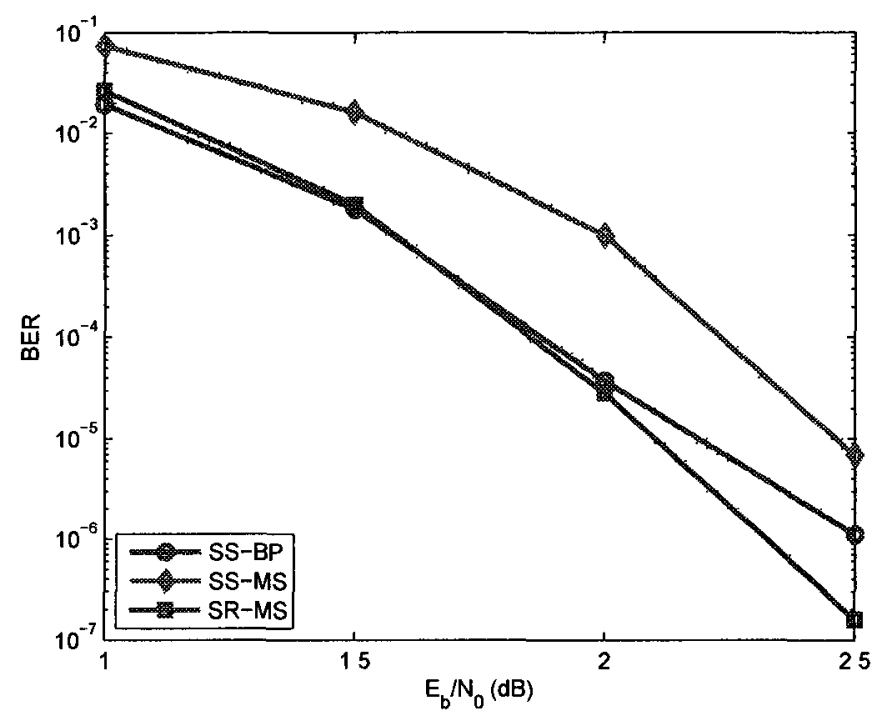

Figure 3.2: BER curves of BP, MS, SR-MS for $(1268,456)$ code.

operation of MS algorithm which is particularly of interest because of its low complexity the performance has shown a considerable improvement. Let us call the SR implementation of MS as SR-MS. Two codes $(1268,456)$ and $(504,252)$ taken from [1i] and [ 14$]$ respectively, have been tested and the simulation results are given in Fig. 3.2 to 3.5. It is assumed that BPSK modulated codewords are transmitted through an AWGN channel and are decoded with three algorithms while the maximum number of iterations is 200 and enough codewords are simulated to generate 100 codeword errors. The performance curves for the BP algorithms are given for reference. The bit error rate curves generated by SR-MS are very close to those of BP algorithm and even better in higher SNRs. The gaps between MS and SR-MS for code $(1268,456)$ and $(504,252)$ are $0.4 \mathrm{~dB}$ and $0.3 \mathrm{~dB}$ respectively, even larger than the gap between BP and MS. Even in terms of the average number of iterations SR-MS is close to other algorithms and they all approach the same value for higher SNRs. Comparing the complexity of BP algorithm with that of SR-MS algorithm, makes SR-MS a very good substitute for BP in fast and real-time applications. The only drawback is the 


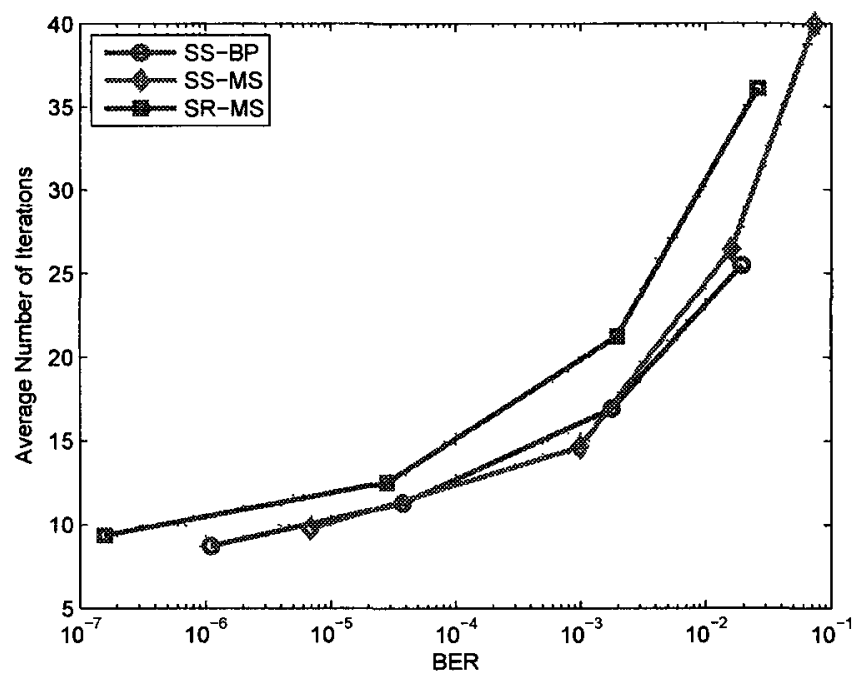

Figure 3.3: Average number of iterations vs. BER for BP, MS, and SR-MS for $(1268,456)$ code.

need for extra memory elements to store the previous variable node messages. Each edge requires its own memory that makes the total of $E=N \times d_{v}$ memories.

Inspired by the idea of memories in the variable nodes we have designed a new binary message passing algorithm for decoding LDPC codes [10,5(1). The algorithm incorporates soft information by storing the quantized received values and differentially updating them in each iteration based on the extrinsic binary messages from the check nodes. While the binary message-passing is particularly attractive for its low complexity and simple implementation (translating to lower area and power consumption in VLSI chips), the incorporation of soft information provides more coding gain and improves the performance. The details of this new algorithm which is a binary implementation of SR-MS algorithm can be found in the following Chapter. 


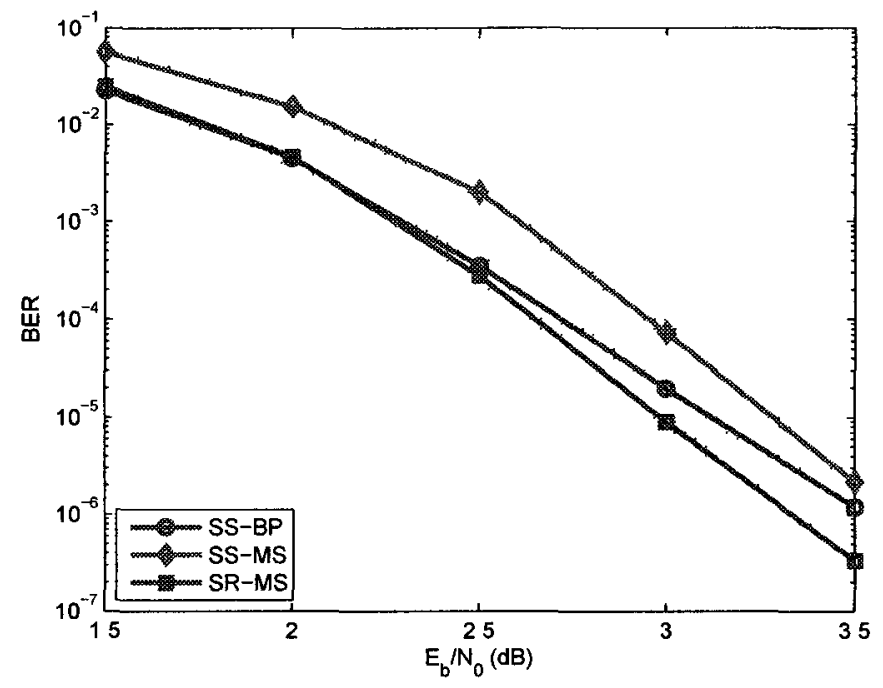

Figure 3.4: BER curves of BP, MS, SR-MS for $(504,252)$ code.

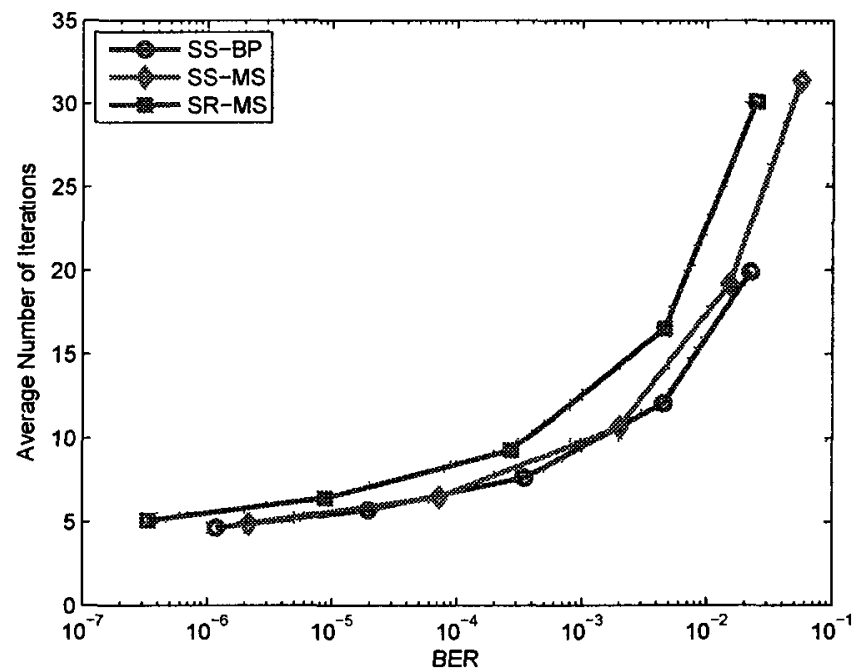

Figure 3.5: Average number of iterations vs. BER for BP, MS, and SR-MS for $(504,252)$ code. 


\section{Chapter 4}

\section{Differential Binary Message Passing Decoding}

In this chapter, we propose a binary message-passing algorithm for decoding lowdensity parity-check (LDPC) codes. The algorithm substantially improves the performance of purely hard-decision iterative algorithms with a small increase in the memory requirements and the computational complexity. We associate a reliability value to each nonzero element of the code's parity-check matrix, and differentially modify this value in each iteration based on the sum of the extrinsic binary messages from the check nodes. For the tested random and finite-geometry LDPC codes, the proposed algorithm can achieve performance as close as $1.3 \mathrm{~dB}$ and $0.7 \mathrm{~dB}$ to that of belief propagation (BP) at the error rates of interest, respectively. This is while, unlike $\mathrm{BP}$, the algorithm does not require the estimation of channel signal to noise ratio. Low memory and computational requirements and binary message-passing make the proposed algorithm attractive for high-speed low-power applications. 


\subsection{Algorithm}

We consider the application of a binary LDPC code $\mathcal{C}$ over a binary-input AWGN channel. Coded bits 0 and 1 are mapped to channel input symbols +1 and -1 , respectively. The channel is memoryless and at the channel output, the received value $y_{\imath}$ corresponding to the transmitted symbol $x_{\imath}$ is given by $y_{\imath}=x_{\imath}+n_{\imath}$, where $n_{\imath}$ is a zero-mean Gaussian noise with variance $\sigma^{2}$ independent of $x_{i}$. Assuming that the AWGN has a power spectral density $N_{0} / 2$, we have $\sigma^{2}=1 /\left(2 R E_{b} / N_{0}\right)$, where $E_{b}$ and $R$ denote the energy per information bit and the code rate, respectively. Suppose that $\mathcal{C}$ is a regular $(n, k)_{\left(d_{v}, d_{c}\right)}$ code with length $n$ described by an $(n-k) \times n$ parity check matrix $\mathbf{H}=\left[\mathrm{H}_{\imath}\right]$, where $d_{v}$ and $d_{c}$ represent the number of ones in each column and each row of $\mathbf{H}$, respectively. They also represent the degrees of variable and check nodes in the Tanner graph of $\mathcal{C}$, respectively.

To implement the algorithm, we consider the received values to be clipped symmetrically at a threshold $c_{t h}$, and then uniformly quantized in the range $\left[-c_{t h}, c_{t h}\right]$. We use the notation $Q\left(y_{\imath}\right)$ to denote the quantized value of $y_{\imath}$. There are $2^{q}-1$ quantization intervals, symmetric with respect to the origin. Each quantization interval has a length $\Delta=\frac{2 c_{t h}}{2^{q}-1}$ and is represented by $q$ quantization bits. Integer numbers $-\left(2^{q-1}-1\right), \cdots, 2^{q-1}-1$ are assigned to the intervals. We associate a $q$-bit memory to each edge in the graph, and initialize it with the quantized received value of the adjacent variable node. The content of this memory is differentially updated in each iteration by being incremented or decremented according to the sum of the extrinsic binary messages of the check nodes. The operations in the check nodes are XOR and the binary message passed from a variable node to a check node is the sign of the memory associated with the edge connecting the two nodes. Decisions are made on the values of variable nodes in each iteration according to the majority of the signs of the adjacent edge-memories and the sign of the initial received value. The decoding 
is stopped if the decisions satisfy the parity-check equations or if a maximum number of iterations $I_{\max }$ is reached.

We use the notations $v_{\imath}, i=1, \cdots, N$, and $c_{\jmath}, j=1, \cdots, M$, to denote the set of variable nodes and the set of check nodes, respectively. The message sent from node $a$ to node $b$ at iteration $\ell$ is denoted by $m_{a \rightarrow b}^{\ell}$. All the messages passed between variable and check nodes are binary with alphabet $\mathcal{A}=\{+1,-1\}$. Notation $B_{v_{\imath}, c_{\jmath}}^{\ell}$ is used for the memory content of the edge adjacent to $v_{\imath}$ and $c_{j}$ at iteration $\ell$. The algorithm is then described by the following steps:

\subsubsection{DD-BMP Algorithm}

1. Initialization, $\ell=0$

$z_{\imath}^{(0)}=\left(1-s g n_{r}\left(y_{\imath}\right)\right) / 2, \quad i=1, \cdots, N$ where $\operatorname{sgn}_{r}(x)=1$, for $x>0$, and $=-1$ for $x<0$. (For $\mathrm{x}=0, \operatorname{sgn}_{r}(x)$ takes +1 or -1 randomly with equal probability.) Let $\boldsymbol{z}^{(0)}=\left(z_{1}^{(0)}, \cdots, z_{N}^{(0)}\right)$. If $\boldsymbol{z}^{(0)} \mathbf{H}^{T}=\mathbf{0}$, the decoding is stopped and the transmitted codeword is estimated by $z^{(0)}$. Otherwise, the memory contents are initiated by $B_{v_{\imath}, c_{\jmath}}^{(0)}=Q\left(y_{\imath}\right), i=1, \cdots, N, \quad j=1, \cdots, M$, for $i$ and $j$ values that satisfy $\mathbf{H}_{\imath \jmath}=1$. The initial messages from variable nodes to check nodes are $m_{v_{\imath} \rightarrow c_{\jmath}}^{(0)}=s g n_{r}\left(B_{v_{\imath}, c_{\jmath}}^{(0)}\right)$. The algorithm is then continued at step 2 .

2. Check Operation, $\ell \geq 1$

$$
m_{c_{\jmath} \rightarrow v_{\imath}}^{(\ell)}=\prod_{v_{k} \in \mathcal{N}\left(c_{\jmath}\right) \backslash v_{\imath}} m_{v_{k} \rightarrow c_{\jmath}}^{(\ell-1)}
$$

where $\mathcal{N}(b)$ denotes the neighboring nodes of node $b$, and $\mathcal{N}(b) \backslash a$ denotes $\mathcal{N}(b)$ excluding $a$. 
3. Variable Operation and Memory Update, $\ell \geq 1$

$$
\begin{gathered}
B_{v_{\imath}, c_{\jmath}}^{(\ell)}=B_{v_{\imath}, c_{\jmath}}^{(\ell-1)}+\sum_{c_{k} \in \mathcal{N}\left(v_{\imath}\right) \backslash c_{\jmath}} m_{c_{k} \rightarrow v_{\imath}}^{(\ell)} \\
m_{v_{\imath} \rightarrow c_{\jmath}}^{(\ell)}=\operatorname{sgn}_{r}\left(B_{v_{\imath}, c_{\jmath}}^{(\ell)}\right)
\end{gathered}
$$

4. Hard-Decision, $\ell \geq 1$

$$
\begin{gathered}
D_{\imath}^{(\ell)}=\sum_{c_{\jmath} \in \mathcal{N}\left(v_{\imath}\right)} \operatorname{sgn}\left(B_{v_{\imath}, c_{\jmath}}^{(\ell)}\right)+\operatorname{sgn}\left(y_{\imath}\right), \\
z_{\imath}^{(\ell)}=\frac{1-\operatorname{sgn}\left(D_{\imath}^{(\ell)}\right)}{2}, \quad i=1, \cdots, N, \quad \text { if } \quad D_{\imath}^{(\ell)} \neq 0
\end{gathered}
$$

where $\operatorname{sgn}(x)=1$, for $x>0 ;=-1$ for $x<0$; and $=0$, for $x=0$. For

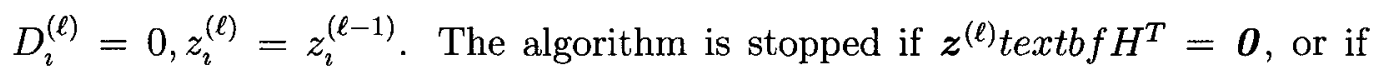
$\ell=I_{\max }$. (In the former case, $z^{(\ell)}$ is used as an estimate for the transmitted codeword, while in the latter case a decoding failure is declared.) Otherwise, $\ell=\ell+1$, and the algorithm is continued from Step 2 .

\subsubsection{Complexity}

To evaluate the complexity of the proposed algorithm, we first consider the number of operations per iteration. In the check nodes, to implement Eq. (1.1) directly, we need to perform $E \times\left(d_{c}-2\right)$ binary XOR operations or binary multiplications, for the $\{0,1\}$ alphabet or the $\{-1,1\}$ alphabet, respectively, where $E=N \times d_{v}$ is the number of edges in the graph. This can be reduced to less than $2 E$ if we first compute Eq. (4.1) over all $\mathcal{N}\left(c_{\jmath}\right)$ and then exclude the term $m_{v_{\imath} \rightarrow c_{\jmath}}^{(\ell-1)}$ for each $v_{\imath}$. In the variable nodes, to implement Eq. (4.2) and (1.3) directly, we need $E \times\left(d_{v}-2\right)$ additions of 
binary values (similar to the check node operations, this can be reduced to less than $2 E$ ), and $E$ binary additions of integer numbers. The hard-decision step is in fact to find the binary value which has the majority among the signs of the memory elements and the sign of the initial value of each variable node. Direct implementation based on Eq. (4.4) requires $E$ binary additions of binary values. In total, the complexity per iteration of the algorithm is about $7 E$ binary operations, many of them involving binary values (bits) or just binary shifts. The algorithm also requires $E$ memory elements, each with $q$ bits.

It should be noted that compared to purely hard-decision algorithms such as majority-based algorithms [3], the complexity per iteration of the proposed algorithm is only slightly higher. Memory requirement however is about $q \times d_{v}$ times that of purely hard-decision algorithms. Compared to soft-decision algorithms such as BP and MS, the complexity per iteration of the proposed algorithm is substantially lower. In addition, compared to the VLSI implementations of soft-decision algorithms with $q$-bit messages, the number of interconnections for the proposed algorithm is smaller by a factor of $q$. This makes the routing much simpler, and would result in shorter interconnections and a smaller chip area. Moreover, since in the VLSI implementation of LDPC decoders the power dissipation of the decoder is largely determined by the switching activity of the wires [:31], the proposed algorithm would significantly reduce the power consumption.

The distribution of the number of iterations plays an important role in the overall complexity of an iterative algorithm and its throughput. It also affects the power consumption of the algorithm in hardware implementations. Our simulation results show that for the tested cases, the average number of iterations for the DD-BMP algorithm is similar to or higher than that of BP and MS algorithms. This will be discussed in more details in the next section. 


\subsection{Simulation results}

One way to estimate the performance of DD-BMP algorithm is application of analytical tools such as density evolution (DE) [3:2] and EXIT charts [; ]. But due to the existence of memory in the algorithm, the analysis will be irrevocably complex. In [ 3$],$ DE analysis of DD-BMP is studied and developed based on truncating the memory of the decoding process and approximating it with a finite order Markov process. The analysis proves the promising performance of DD-BMP algorithm in general. But in the case of FG codes or high rate codes the complexity of the analysis makes it intractable due to inadequacy of computational resources such as memory and CPU time. In this cases the only solution is to estimate the performance through simulations.

To study the performance of the DD-BMP algorithm, we use Monte Carlo simulations. Using simulations, we have tested a large number of codes with different rates, block lengths and degree distributions. We have also tested both randomly constructed and finite-geometry (FG) codes. In the following, we report our results for eleven codes: eight randomly constructed regular, one irregular and two FG codes. The randomly constructed codes are taken from [ $\mid Y]$ and are divided into three groups. The first group consists of three codes with the degree distribution of $\left(d_{v}, d_{c}\right)=(4,6)$ and different lengths. The second group contains four $(n, k)=(4000,2000)$ codes with different degree distributions. And the third group holds only one high rate $(1057,813)_{(3,13)}$ code. The irregular $(1008,504)$ code has been specifically designed with the following degree distribution for BP algorithm in [; 1$]$ : $\rho(x)=0.002 x^{6}+0.9742 x^{7}+0.0238 x^{8}$ representing variable node degree distribution, and $\lambda(x)=0.4772 x+0.2808 x^{2}+0.0347 x^{3}+0.0962 x^{4}+0.0089 x^{6}+0.001 x^{13}+0.1012 x^{14}$ check node degree distribution. Where $x^{k}$ represents a node with degree $k$ and the 
corresponding coefficient indicates the ratio of nodes with degree $k$ in the code. Finally, the FG codes have parameters $(273,191)_{(17,17)}$ and $(1023,781)_{(32,32)}$. For all the simulations the maximum number of iterations is set to 100 and for each simulation point, 100 code word errors are found.
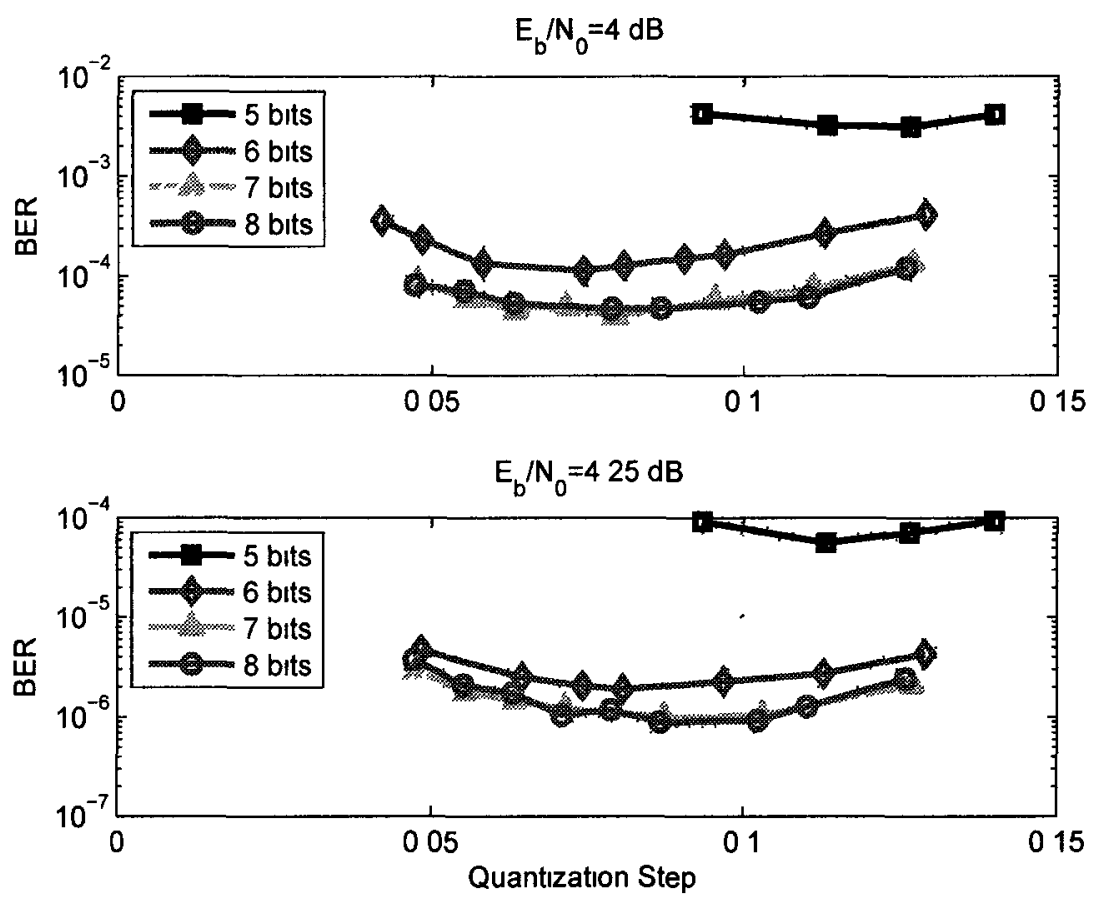

Figure 4.1: BER vs. $\triangle$ for different values of $q$ at SNR values $4 \mathrm{~dB}$ and $4.25 \mathrm{~dB}$ for code $(3000,1000)_{(4,6)}$.

Our simulation results show that the optimal quantization step $\triangle^{*}$, which minimizes the BER, is rather independent of the SNR, and changes only slightly with the number of quantization bits $q$ for larger values of $q$. This is demonstrated for $(3000,1000)_{(4,6)}$ in Fig. 41 , where the BER is plotted vs. $\triangle$ for different values of $q$ at SNR values $4 \mathrm{~dB}$ and $4.25 \mathrm{~dB}$. Fig. 41 also shows that by increasing $q$, the performance improves until it reaches a saturation point beyond which the improvement in performance is very small. For all codes, the optimal values of $\Delta^{*}$ and $q$ are 
found and are reported in Table 1.1. These values appear to strike the right balance between the performance and the complexity. It can be seen from Table 4.1 that for the most of the randomly constructed codes optimal $q$ is 7 bits and only those codes with variable node degrees smaller than 4 can reach desired performance with fewer bits.

Table 4.1: Optimal values of $q$ and $\triangle^{*}$ for different codes

\begin{tabular}{c|c|c|c|c}
\hline Code & $(n, k)$ & $\left(d_{v}, d_{c}\right)$ & $\triangle^{*}$ & $q$ \\
\hline \hline 1 & $(3000,1000)$ & $(4,6)$ & 0.0952 & 7 \\
2 & $(6000,2000)$ & $(4,6)$ & 0.0952 & 7 \\
3 & $(12000,4000)$ & $(4,6)$ & 0.0952 & 7 \\
4 & $(4000,2000)$ & $(6,12)$ & 0.0157 & 8 \\
5 & $(4000,2000)$ & $(5,10)$ & 0.027 & 7 \\
6 & $(4000,2000)$ & $(4,8)$ & 0.0476 & 7 \\
7 & $(4000,2000)$ & $(3,6)$ & 0.2 & 5 \\
8 & $(1057,813)$ & $(3,13)$ & 0.1613 & 6 \\
9 & $(1008,504)$ & Irr & 0.1452 & 6 \\
10 & $(273,191)$ & $(17,17)$ & 0.0222 & 7 \\
11 & $(1023,781)$ & $(32,32)$ & 0.0043 & 9 \\
\hline
\end{tabular}

The BER curves of DD-BMP are given in Figures 1.2 to 1.7 . In the figures, we have also given the performance curves for BP, MS and MB algorithms for reference. For each code, the MB algorithm with the best performance is selected. For all given regular codes, GA has the best performance (threshold) among all the MB algorithms [.:1]. For $(273,191)_{(17,17)}$ and $(1023,781)_{(32,32)}, \mathrm{MB}^{3}$ and $\mathrm{MB}^{5}$ are used, respectively. The results show that for all the codes the performance of DD-BMP is substantially better than that of MB algorithms. For random codes, the performance gap is as large as about $4 \mathrm{~dB}$ at higher SNR values, while this gap is reduced to 


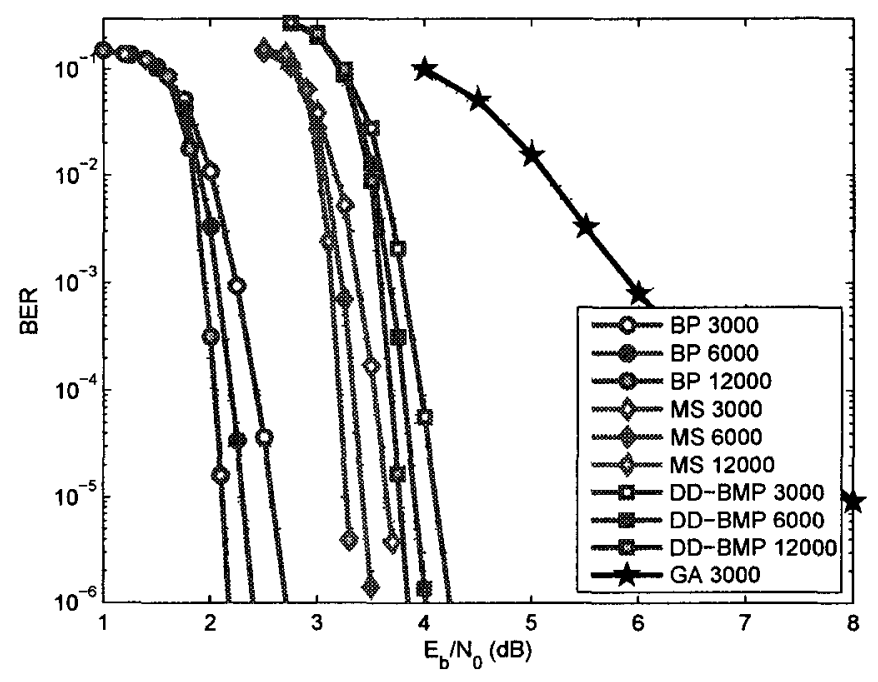

Figure 4.2: BER curves of BP, MS, DD-BMP, and GA for codes $(3000,1000)_{(4,6)}$, $(6000,2000)_{(4,6)}$ and $(12000,4000)_{(4,6)}$

up to about $1.5 \mathrm{~dB}$ for the FG codes. In particular for some regular codes, the GA algorithm demonstrates an early error flare while DD-BMP does not. Similar trends are observed for the word error rate curves.

Compared to soft-decision algorithms, the results show that for all the random codes, the performance of DD-BMP is within $1.9 \mathrm{~dB}$ of the $\mathrm{BP}$ performance. For the FG codes, the performance gap between DD-BMP and BP is much smaller, and is about $0.7 \mathrm{~dB}$ at high SNR values. The performance of DD-BMP for all random codes is at most $0.4 \mathrm{~dB}$ inferior to that of MS except for $(1057,813)_{(3,13)}$ for which the gap is larger and about $1.9 \mathrm{~dB}$, since for this code, BP and MS have almost identical performance for large values of SNR. For FG codes, however, the performance of DD-BMP is superior to that of MS by about $0.2 \mathrm{~dB}$ and $0.5 \mathrm{~dB}$, for $(273,191)_{(17,17)}$ 


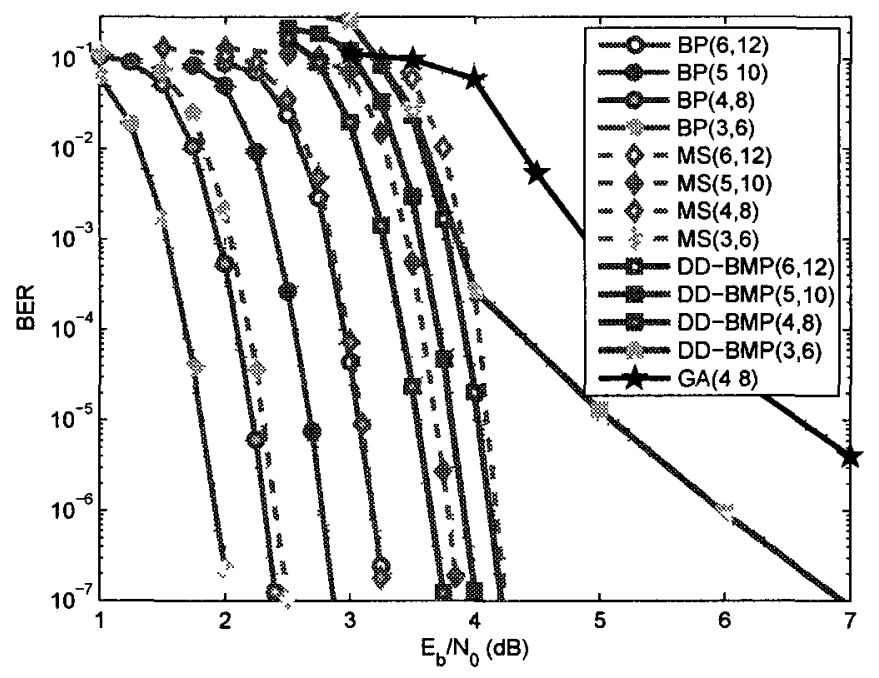

Figure 4.3: BER curves of BP, MS, DD-BMP, and GA for code $(4000,2000)$ with different degree distributions.

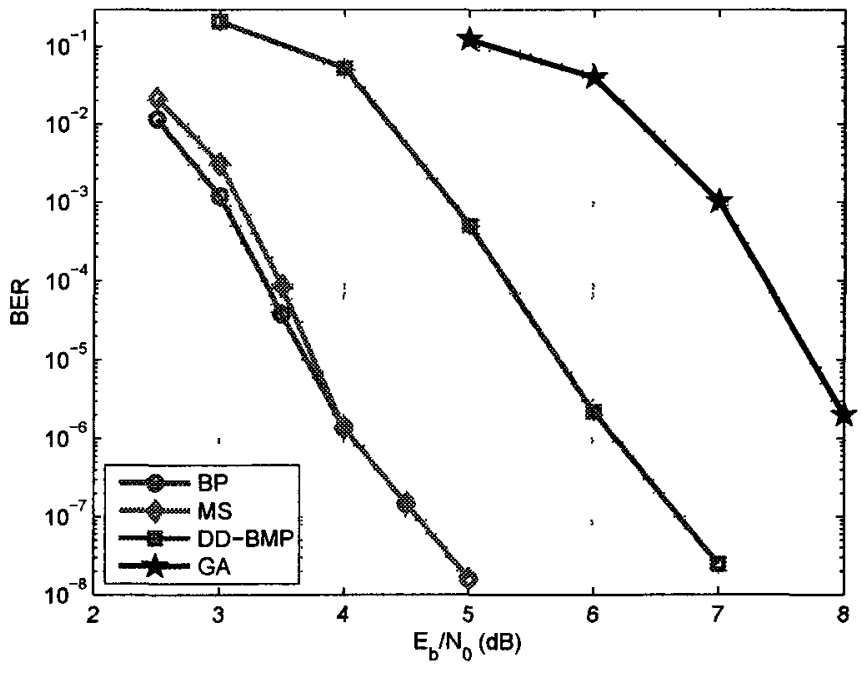

Figure 4.4: BER curves of BP, MS, DD-BMP, and GA for code $(1057,813)_{(3,13)}$. 


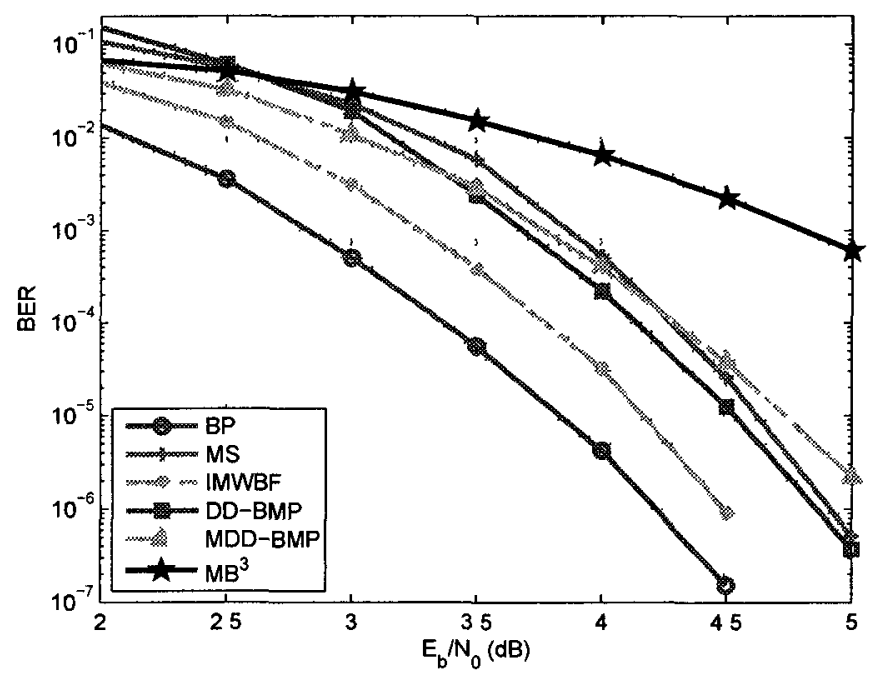

Figure 4.5: BER curves of BP, MS, IMWBF, DD-BMP, MDD-BMP and GA for code $(273,191)_{(17,17)}$.

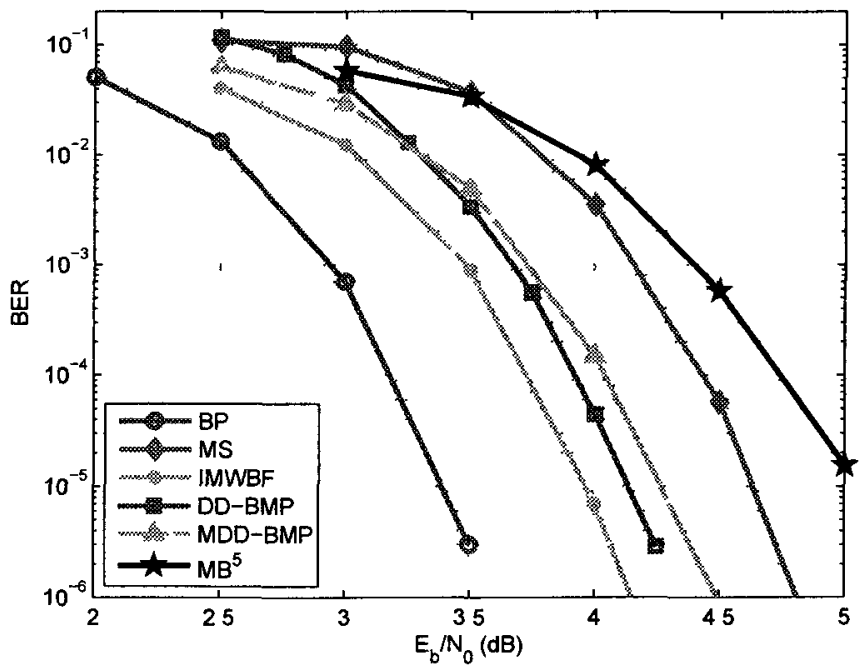

Figure 4.6: BER curves of BP, MS, IMWBF, DD-BMP, MDD-BMP and GA for code $(1023,781)_{(32,32)}$. 


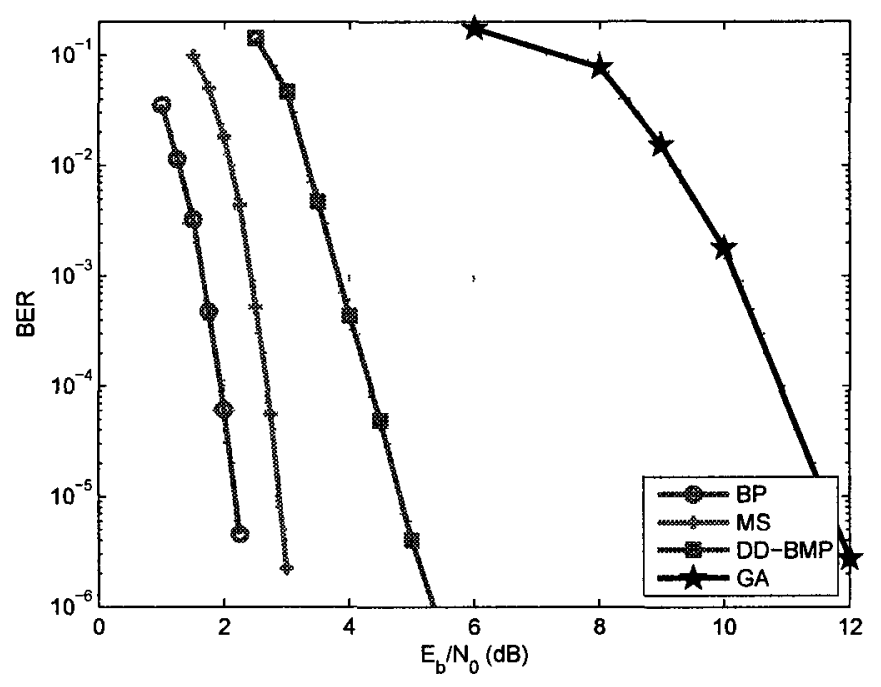

Figure 4.7: BER curves of BP, MS, DD-BMP, and GA for irregular code $(1008,504)$. and $(1023,781)_{(32,32)}$, respectively.

In Fig. 12, the BER curves of DD-BMP for randomly constructed $(3000,1000)_{(4,6)},(6000,2000)_{(4,6)}$ and $(12000,4000)_{(4,6)}$ codes are plotted. These codes have the same degree distribution and thus the same $\triangle^{*}$. The comparison of the three curves shows a threshold phenomenon. Threshold values for DD-BMP algorithm can be found in $\left[\begin{array}{ll}3 \\ 1\end{array}\right]$.

To further study the effect of degree distributions on the performance of DDBMP, we have tested this algorithm on a $(4000,2000)$ code with different variable node degrees of $3,4,5$, and 6 . The BER results are reported in Fig. 43 . These results show that for the same block length and rate, the performance of DD-BMP degrades as the variable node and the check node degrees increase. It is important to note that the trend for both $\mathrm{BP}$ and MS algorithms is exactly the same, i.e., the performance of both BP and MS degrades as $d_{v}$ and $d_{c}$ are increased for a rate $\frac{1}{2}$ regular code [ $\left[\begin{array}{l}32] \\ 2\end{array}\right.$. This can also be observed in Fig. 13, where we have added the BER curves of both BP and MS algorithms for the two codes. A careful inspection 
of Fig. 4.3 shows that the performance gap between BP and MS on one hand and DD-BMP on the other hand is decreased as $\left(d_{v}, d_{c}\right)$ is increased from $(4,8)$ to $(5,10)$ and $(6,12)$. In fact, for the $(6,12)$ code, the performance difference between MS and DD-BMP reverses and DD-BMP outperforms MS. In Fig. 1.3, we have also presented the BER for a $(4000,2000)_{(3,6)}$ code under DD-BMP, BP and MS. It can be seen that this degree distribution violates the trends just explained for DD-BMP. In particular, the BER curve for this code demonstrates an early error flare. We have tested a $(8000,4000)_{(3,6)}$ code as well, and the same behavior is also observed for that code. All the above observations closely follow the analytical analysis reported in [5.3] Table II.

Finally, DD-BMP can also be applied to irregular LDPC codes. In Fig. 4.7, we have provided the BER curves of DD-BMP, BP and MS for a $(1008,504)$ irregular code with the degree distribution optimized for BP and the code is constructed using the modified PEG construction of [3/]. As can be seen it has reduced the gap between GA and BP algorithm. But at high bit error rates it is still $3 \mathrm{~dB}$ away from $\mathrm{BP}$ algorithm. In terms of convergence DD-BMP needs a higher average number of iterations in comparison to other decoding algorithms.

The average number of iterations vs. BER for BP, MS, DD-BMP and MB algorithms are given in Figures 4.8 to 4.13 for all codes. These figures show that the average number of iterations for DD-BMP is in general larger than those of the other algorithms. The difference however is smaller, and in the cases of $(1057,813)_{(3,13)}$ and $(273,191)_{(17,17)}$ is negligible, for lower BER values.

WBF algorithms and their variants and modifications $[30,1,1,3,3 y]$ have proved 


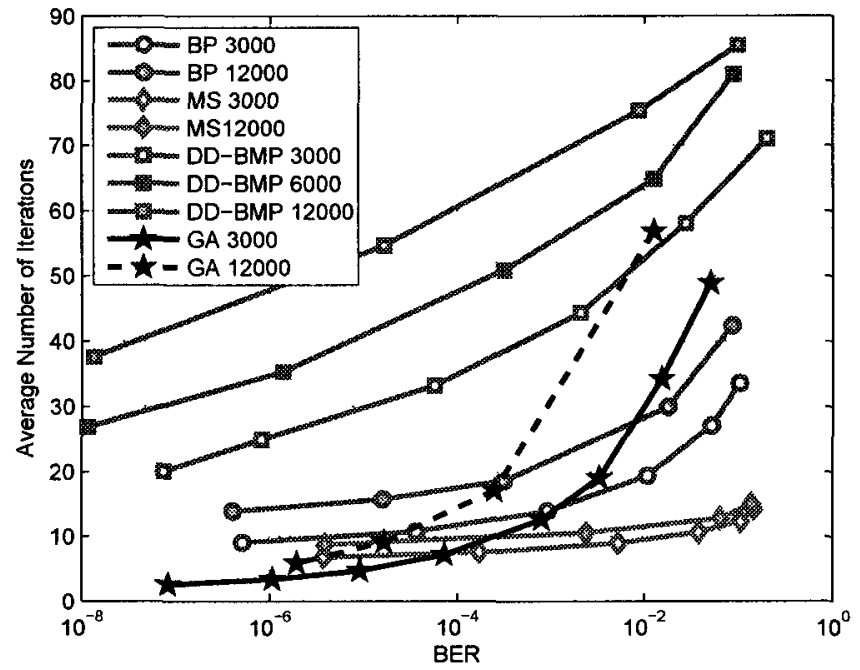

Figure 4.8: Average number of iterations vs. BER for BP, MS, DD-BMP and GA for code $(3000,1000)_{(4,6)},(6000,2000)_{(4,6)}$ and $(12000,4000)_{(4,6)}$.

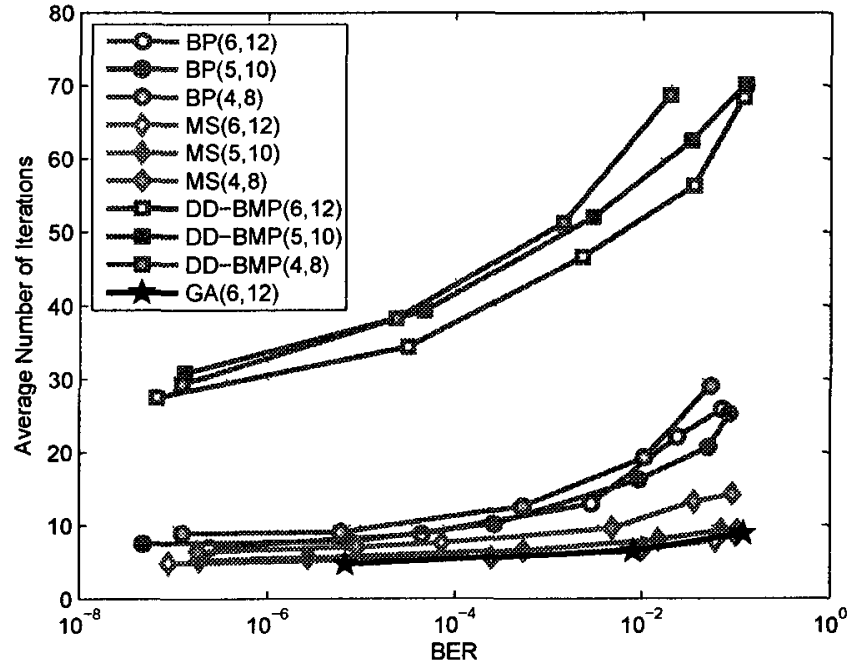

Figure 4.9: Average number of iterations vs. BER for BP, MS, DD-BMP and GA for code $(4000,2000)$ with different degree distributions. 


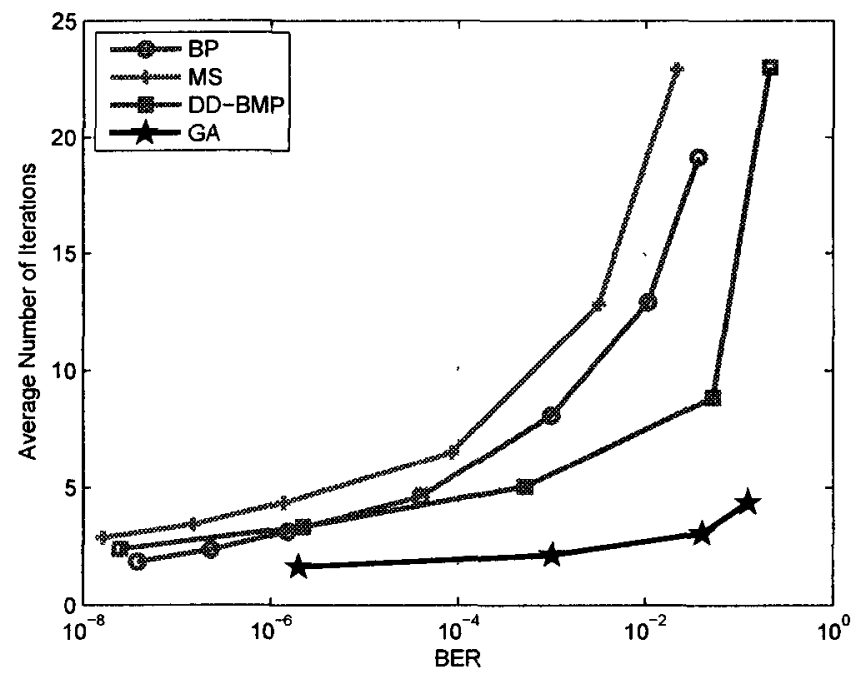

Figure 4.10: Average number of iterations vs. BER for BP, MS, DD-BMP, and GA for code $(1057,813)_{(3,13)}$.

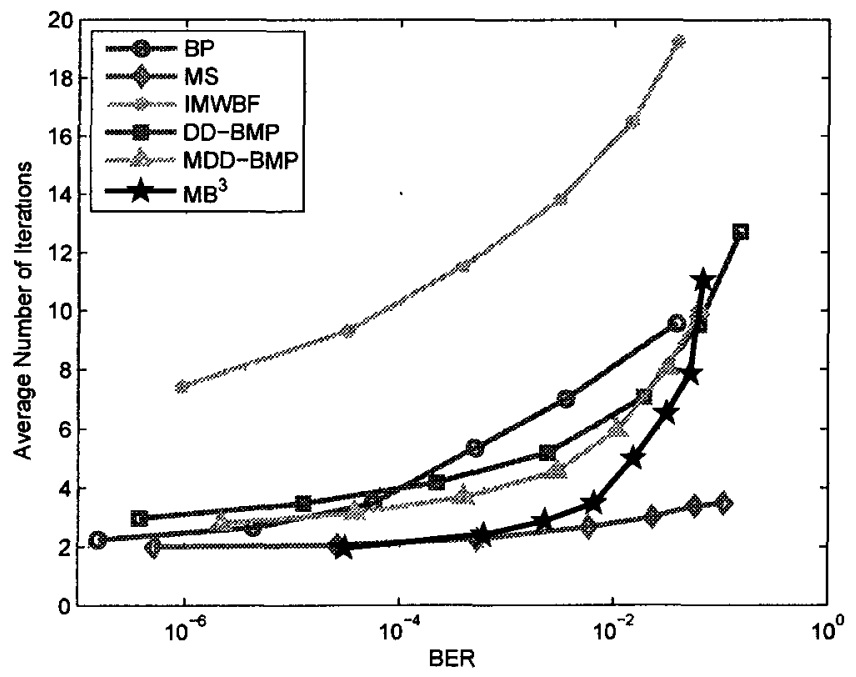

Figure 4.11: Average number of iterations vs. BER for BP, MS, IMWBF, DD-BMP, MDD-BMP and GA for code $(273,191)_{(17,17)}$. 


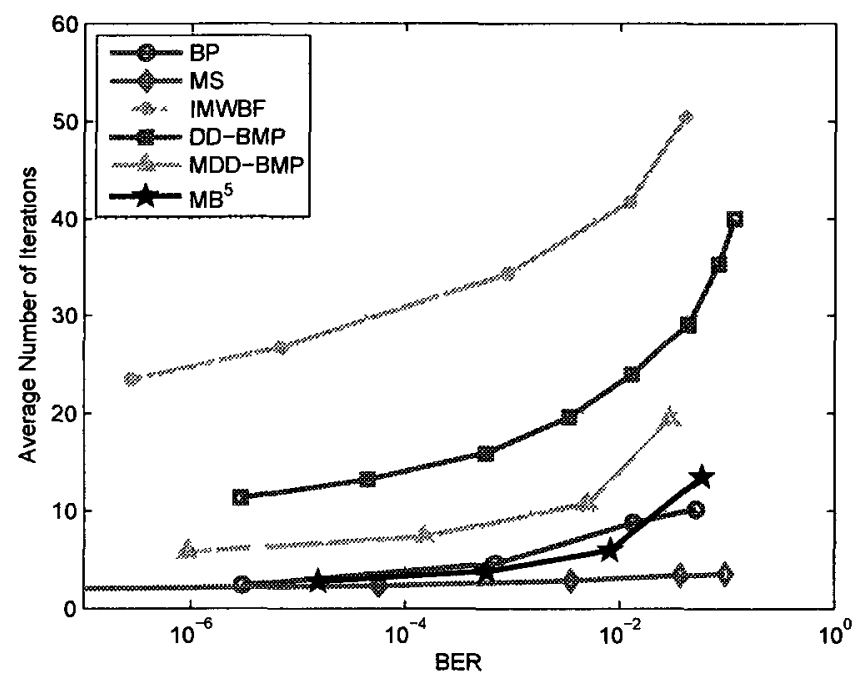

Figure 4.12: Average number of iterations vs. BER for BP, MS, IMWBF, DD-BMP, MDD-BMP and GA for code $(1023,781)_{(32,32)}$.

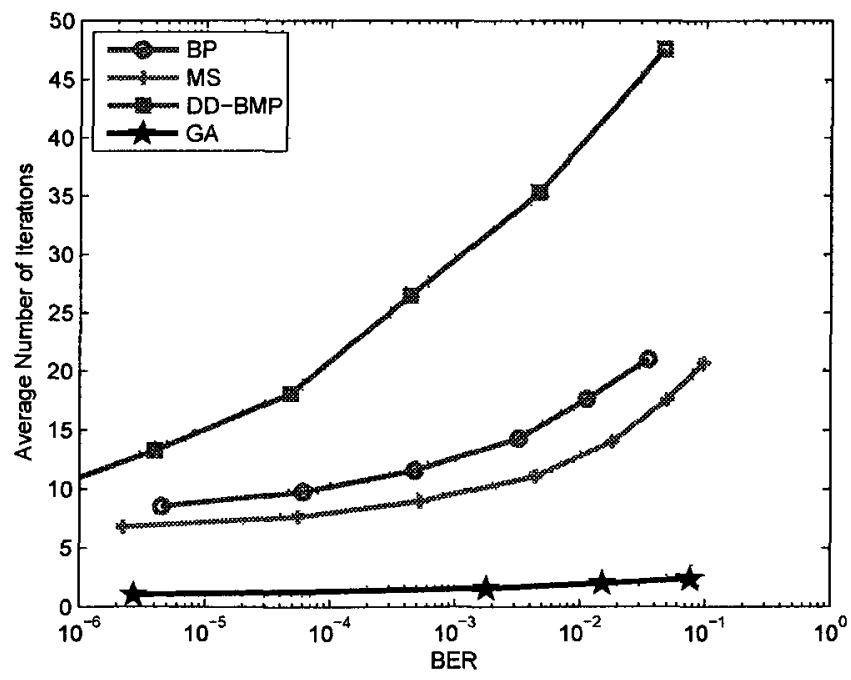

Figure 4.13: Average number of iterations vs. BER for BP, MS, DD-BMP, and GA for irregular code $(1008,504)$. 
to perform well for decoding FG codes. The best performing algorithm in this category is the improved modified WBF (IMWBF) algorithm of [30]. The performance of this algorithm is given in Figures 4.5 and 4.6 for $(273,191)_{(17,17)}$ and $(1023,781)_{(32,32)}$, respectively. As can be seen, IMWBF slightly outperforms DD-BMP by about $0.4 \mathrm{~dB}$ and $0.2 \mathrm{~dB}$ for $(273,191)_{(17,17)}$ and $(1023,781)_{(32,32)}$, respectively. This improvement in performance however is counter-balanced by the much larger average number of iterations as illustrated in Figures 4.11 and 4.12 , for $(273,191)_{(17,17)}$ and $(1023,781)_{(32,32)}$, respectively. It is also important to note that all the stored and computed values in IMWBF are floating-point real numbers. One would expect the performance to deteriorate for a fixed-point implementation of IMWBF. For random codes, DD-BMP usually outperforms IMWBF handily. From the computational complexity point of view, IMWBF requires about $2 E$ real comparisons, $E$ real additions and $N$ real multiplications for preprocessing before each iterative decoding to compute the reliability information of check nodes and the initial values of the flipping functions. During each iteration, IMWBF needs about $d_{v} \times d_{c}$ real additions and $N$ real comparisons. It also requires $2 M$ real memory elements to store the reliability information of the check nodes.

It is worth mentioning that we have also tried simplified variants of DD-BMP algorithm. In particular we have tested a version of the algorithm where the memory update operation of Eq. (1.2) is modified to

$$
B_{v_{i}, c_{j}}^{(\ell)}=B_{v_{i}, c_{j}}^{(\ell-1)}+\operatorname{sgn}\left(\sum_{c_{k} \in \mathcal{N}\left(v_{i}\right) \backslash c_{j}} m_{c_{k} \rightarrow v_{i}}^{(\ell)}\right)
$$

It should be noted that Eq. (4.6) reduces to Eq. (4.2) for $d_{v}=3$. In general, the performance of this algorithm appears to be only slightly inferior to that of the original algorithm. The advantage however is the lower complexity as the operation in Eq. (4.6) can be performed by a count up/down of the memory. 
Another variant of the algorithm is to assign a memory to each variable node instead of each edge in the graph and update its content in every iteration based on the binary messages of the check nodes. The outgoing messages from a variable node are all identical and are the sign of the memory content. This algorithm is simpler and requires less memory but the performance for random codes is considerably worse than that of the original algorithm. For FG codes the performance loss is smaller as can be seen in Figures 4.5 and 4.6. In these figures the performance of this algorithm is labeled as MDD-BMP, brief for modified DD-BMP. The average number of iterations vs. BER for MDD-BMP is also given in Figures 4.11 and 4.12. As can be seen, at lower BER values, MDD-BMP has a slight advantage over DD-BMP in terms of average number of iterations. 


\section{Chapter 5}

\section{Adaptive Belief Propagation Decoding of LDPC Codes}

An adaptive control technique, originally presented by Kocarev et al. [36] to improve the performance of turbo codes in the waterfall region, is applied to low-density parity-check (LDPC) codes. For typical finite-length LDPC codes, the application of the proposed technique improves the performance of belief propagation (BP) by up to about $0.5 \mathrm{~dB}$ in the waterfall region. The proposed technique also improves the performance of $\mathrm{BP}$ in the error floor region significantly. This is in contrast with the results for turbo codes, where the technique appears to have no effect on the error floor. We also show that the technique is robust against the changes of the channel signal-to-noise ratio (SNR) and that the same control parameters can be used over a wide range of SNR values with negligible performance degradation.

Belief propagation (BP) is widely used for decoding low-density parity-check (LDPC) [l] and turbo [5] codes. Applied to cycle free graphs, which represent codes with infinite block length, BP converges to a posteriori probabilities for bits. For practical codes, where the graph representation has many small cycles, BP, although still performing very well, becomes suboptimal [11]. The sub-optimality can 
be attributed to the over-estimation of the reliability of messages [11], and the performance of $\mathrm{BP}$ for finite-length codes can be enhanced by scaling or offsetting down the reliability of messages $[11,7, \cdots, 7]$.

Inspired by the representation of iterative decoding algorithms as nonlinear dynamical systems, Kocarev et al. proposed an alternate technique to improve the performance of BP for finite-length turbo codes [36i], [- The technique, which is based on the adaptive control of transient chaos, is capable of improving the performance of turbo decoders by 0.2 to $0.3 \mathrm{~dB}$ in the waterfall region [36], [5] The performance in the error floor region however remains unaffected. This is justified by the lack of transient chaos in this region [30]. While the technique of $[36,50]$ is similar to those proposed in $[11,20]$ and $[\because]$ as it also aims at reducing the reliability of messages, it also differs from them in that the reduction in reliability of a message is achieved adaptively by a correction factor which is a function of the message itself.

LDPC codes, although similar to turbo codes in that they also belong to the category of iterative coding schemes, sometimes demonstrate behaviors very different than those of turbo codes. One example is the application of successive relaxation (SR) to the iterative decoding of LDPC and turbo codes. While for turbo codes, SR does not provide any improvement over the conventional successive substitution (SS) [11], for LDPC codes significant improvements are observed [1;3]. We demonstrate another example of different behavior of turbo codes and LDPC codes in this thesis. We apply the adaptive control technique of $[3$, , n the BP decoding of LDPC codes. Using simulations, we demonstrate performance improvements of about $0.5 \mathrm{~dB}$ in the waterfall region for the tested LDPC codes. Moreover, our results show significant performance improvement in the error floor region. This is in stark contrast with the results of turbo codes $[\because, 1,-18]$, where the technique has almost no effect on the error floor. This motivates us to analyze the performance of the technique in the error floor region. To carry out the analysis, we use the a posteriori average entropy [06] as 
a simple representation of decoding trajectories, and show that the improvement in the error floor performance is a direct result of improvement in the chaotic transient behavior of the decoder.

\subsection{System Model and the Adaptive Control Tech- nique}

Consider the application of a binary LDPC code $\mathcal{C}$ over a binary-input AWGN channel such that coded bits 0 and 1 are mapped to the channel input symbols +1 and -1 , respectively. Assume that $\mathcal{C}$ has length $N$ and is described by an $M \times N$ parity check matrix $\mathbf{H}=\left[\mathbf{H}_{m n}\right]$. For a regular $\left(d_{v}, d_{c}\right)$ code, parameters $d_{v}$ and $d_{c}$ represent the number of ones in each column and each row of $\mathbf{H}$, respectively. They also represent the degrees of variable and check nodes in the Tanner graph [27] of $\mathcal{C}$, respectively. For irregular codes, not all the rows or all the columns have the same weight.

Suppose that the channel output $y_{i}$ corresponding to the transmitted symbol $x_{i}$ is given by $y_{i}=x_{i}+n_{i}$, where $n_{i}$ is a zero-mean Gaussian noise with variance $\sigma^{2}$ independent of $x_{i}$. Assuming that the AWGN has a power spectral density $N_{0} / 2$, we have $\sigma^{2}=1 /\left(2 R E_{b} / N_{0}\right)$, where $E_{b}$ and $R$ denote the energy per information bit and the code rate, respectively. We consider a parallel BP decoder implemented in the log-likelihood ratio (LLR) domain. The input to the decoder for the ith bit is the channel LLR $2 y_{i} / \sigma^{2}$. The decoder performs iterations until it converges to a codeword or a maximum number of iterations $I_{\max }$ is reached.

To apply the adaptive control technique, we replace the outgoing messages $\mathbf{m}_{v \rightarrow c}$ of variable nodes in the original $\mathrm{BP}$ algorithm by $[\because, ;, ;]$

$$
\mathbf{m}_{v \rightarrow c}^{\prime}=\alpha \mathbf{m}_{v \rightarrow c} e^{-\beta\left|\mathbf{m}_{v \rightarrow c}\right|}
$$




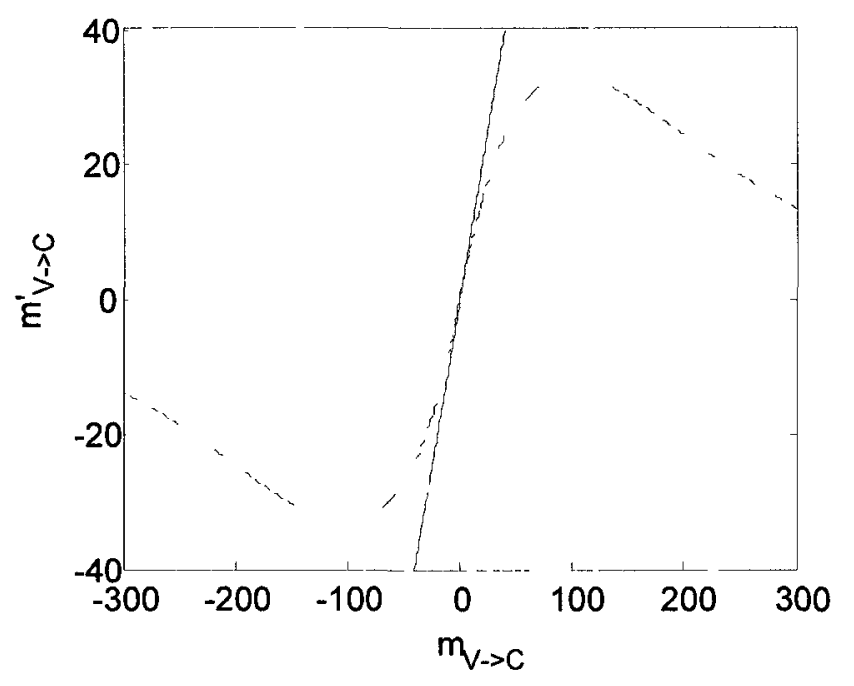

Figure 5.1: An example of Eq. (5.1).

The control function is chosen because if $x_{\imath}$ is small, then the attenuation factor in (5.1) is close to 1 (since $\alpha$ is close to 1 and $\beta$ is small). In other words, the control algorithm does nothing. If, however, $x_{\imath}$ is large, then the control algorithm reduces the normalization factor, thereby attenuating the effect of $x_{\imath}$ on the decoding algorithm. Fig. 5.1 shows a realization of Eq. (5.1), as can be seen in this figure the function's slope for small values is close to 1 .

where $v$ and $c$ are a pair of neighboring variable and check nodes, respectively, and parameters $\alpha$ and $\beta$ are constants selected from the intervals $(0,1]$ and $[0,+\infty)$, respectively, to optimize the error rate performance of the algorithm. The optimal values of $\alpha$ and $\beta$ are usually close to the end and the beginning of the corresponding intervals, respectively. It should be noted that the normalized BP algorithm of [1 I] is a special case of Eq. (5.1) with $\beta=0$, i.e., no adaptation. We have also applied the adaptation to the output of check nodes as well as both the outputs of variable nodes and check nodes. In both cases, the results are similar to the results reported 
here.

\subsection{Simulation Results and Analysis}

To study the effects of the adaptive control technique on the BP decoding of finitelength LDPC codes, we consider an optimized $(1268,456)$ irregular code, also used in [11], and a regular $(504,252)$ code from [ 4 . For all the simulations, the maximum number of iterations is set to 200 and for each simulation point, 100 codeword errors are generated.

For the $(1268,456)$ and $(504,252)$ codes, the optimal values of $\alpha$ and $\beta$, which minimize the bit error rate (BER), are found to be 1 and 0.008 , and 0.99 and 0.010 , respectively. These values which are obtained by exhaustive search at $E_{b} / N_{0}=2.5$ $\mathrm{dB}$ and $4 \mathrm{~dB}$ for the two codes, respectively, remain close-to-optimal over a wide range of SNR values of interest. Fig. 5.2 shows BER curves of both codes decoded by the original $\mathrm{BP}$, and $\mathrm{BP}$ with adaptive control. We have also included in Fig. 5.2 the BER curves for normalized BP. The value of a for normalized BP, optimized for the $(1268,456)$ and $(504,252)$ codes at $E_{b} / N_{0}=2.5 \mathrm{~dB}$ and $4 \mathrm{~dB}$, is 0.9 and 0.75 , respectively. As can be seen, $\mathrm{BP}$ with adaptive control outperforms standard BP by about $0.25 \mathrm{~dB}$ to $0.6 \mathrm{~dB}$ for $\mathrm{BER}$ values in the range of $10^{-7}-10^{-6}$. Adaptive $\mathrm{BP}$ also outperforms non-adaptive normalized BP. In particular, for the $(1268,456)$ code, the error floor performance of the former is superior to the latter. For the $(504,252)$ code, the performance improvement is mainly happening at lower SNR values. The reason for this is the high sensitivity of the optimal value of $\alpha$ to the variations of SNR for normalized BP. For example, for the $(504,252)$ code while optimal $\alpha$ is 0.75 at $E_{b} / N_{0}=4 \mathrm{~dB}$, it increases to 0.95 at $2.0 \mathrm{~dB}$. This sensitivity is substantially reduced by the introduction of adaptive control in the algorithm of Eq. (5.1).

Fig. 5.3 compares the average number of iterations of the original BP, BP with 
adaptive control and normalized BP versus BER for the two codes. The figure shows that the improvement in performance for adaptive BP comes at no cost in the average number of iterations. In fact, for lower $\mathrm{SNR}$ values, adaptive $\mathrm{BP}$ requires a smaller average number of iterations to converge compared to Normalized BP for both codes. Compared to standard BP, there is an improvement at low SNR region for the $(504,252)$ code.

To analyze the superior performance of adaptive BP, we use the a posteriori average entropy defined by $[; ; 0]$

$$
\mathcal{E}(i)=-\frac{1}{N} \sum_{\jmath=1}^{N}\left(p_{\jmath}^{0}(i) \log _{2} p_{\jmath}^{0}(i)+p_{\jmath}^{1}(i) \log _{2} p_{\jmath}^{1}(i)\right)
$$

where $i$ is the iteration number, and $p_{\jmath}^{k}(i), k \in\{0,1\}$ is the a posteriori probability of the $j$ th bit being equal to $k$ in iteration $i$. Although $\mathcal{E}(i)$ is probably too simple to provide a comprehensive picture of the dynamical behavior of the decoder, its evolution with $i$ can be effectively used to identify the type of error events in which the decoder is trapped. When the decoder converges to the right codeword, we have $\mathcal{E}=0$. Fig. $5.4(\mathrm{~b})$ shows the steady-state evolution of $\mathcal{E}(i+1)$ vs. $\mathcal{E}(i)$ for three instances of iterative decoding, which we associate with fixed-pattern, periodicpattern and random-like or chaotic error events. For the three instances, the number of decoding errors versus the iteration number is also plotted in Fig. 5.4(a).

To analyze the difference in performance of standard BP and adaptive BP at the high SNR region, we examine and categorize the failures of both algorithms and track the changes with the increase in the maximum number of iterations $I_{\text {max }}$. In particular, for the $(1268,456)$ code and the $(504,252)$ code, we present the results at $E_{b} / N_{0}=2.5 \mathrm{~dB}, 2.75 \mathrm{~dB}, 3 \mathrm{~dB}$, and $3.5 \mathrm{~dB}, 4 \mathrm{~dB}, 4.5 \mathrm{~dB}$, in Tables 5.1 and 5.2 , 

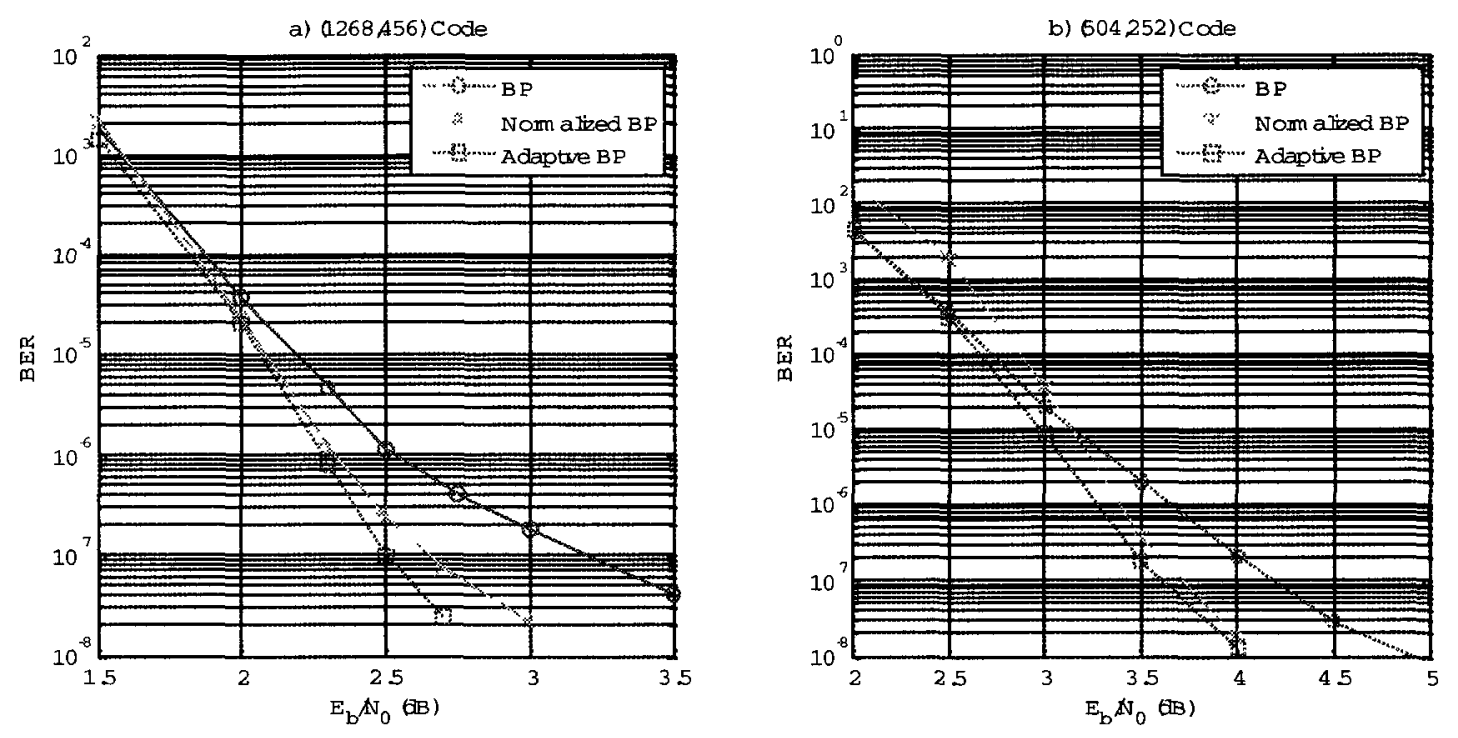

Figure 5.2: BER for a) $(1268,456)$ and b) $(504,252)$ LDPC codes decoded by standard BP, Normalized BP, and adaptive BP algorithms.
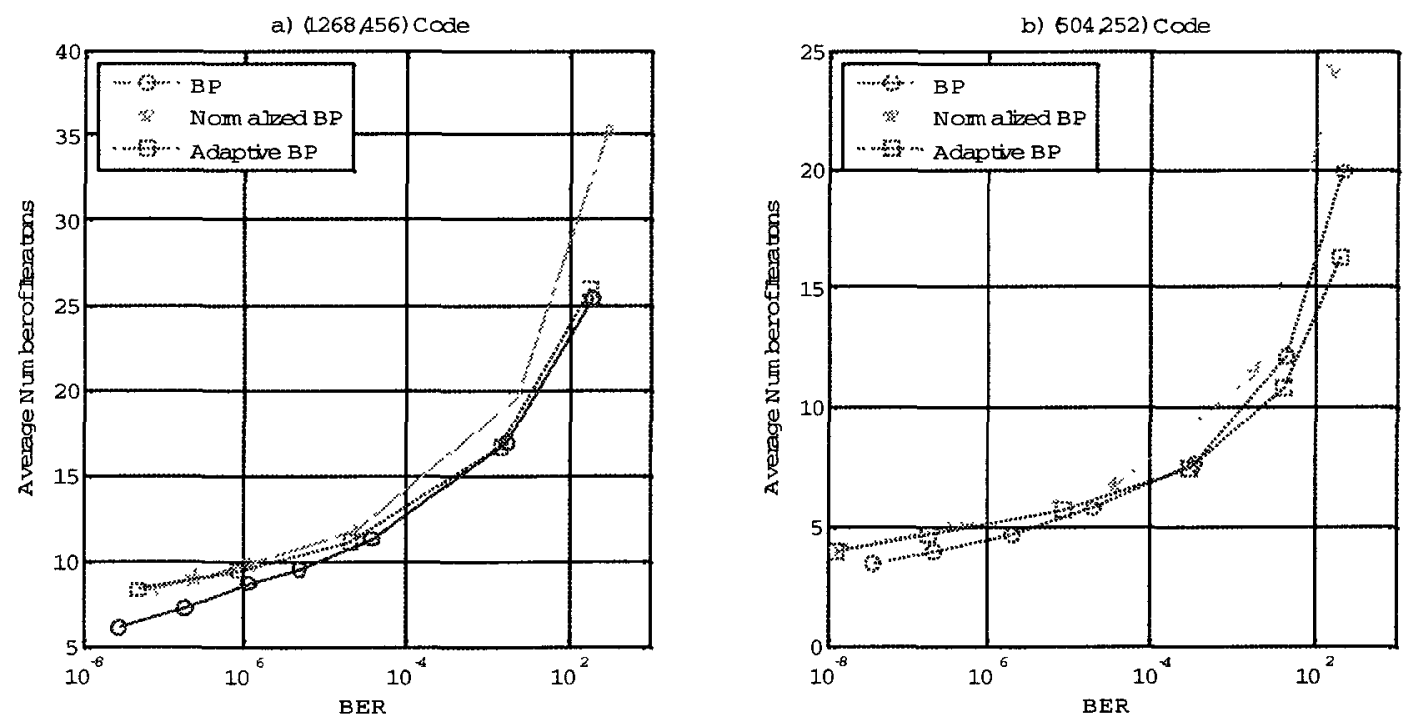

Figure 5.3: Average number of iteration versus BER for a) $(1268,456)$ and b) $(504,252)$ LDPC codes decoded by standard BP, Normalized BP, and adaptive BP algorithms. 

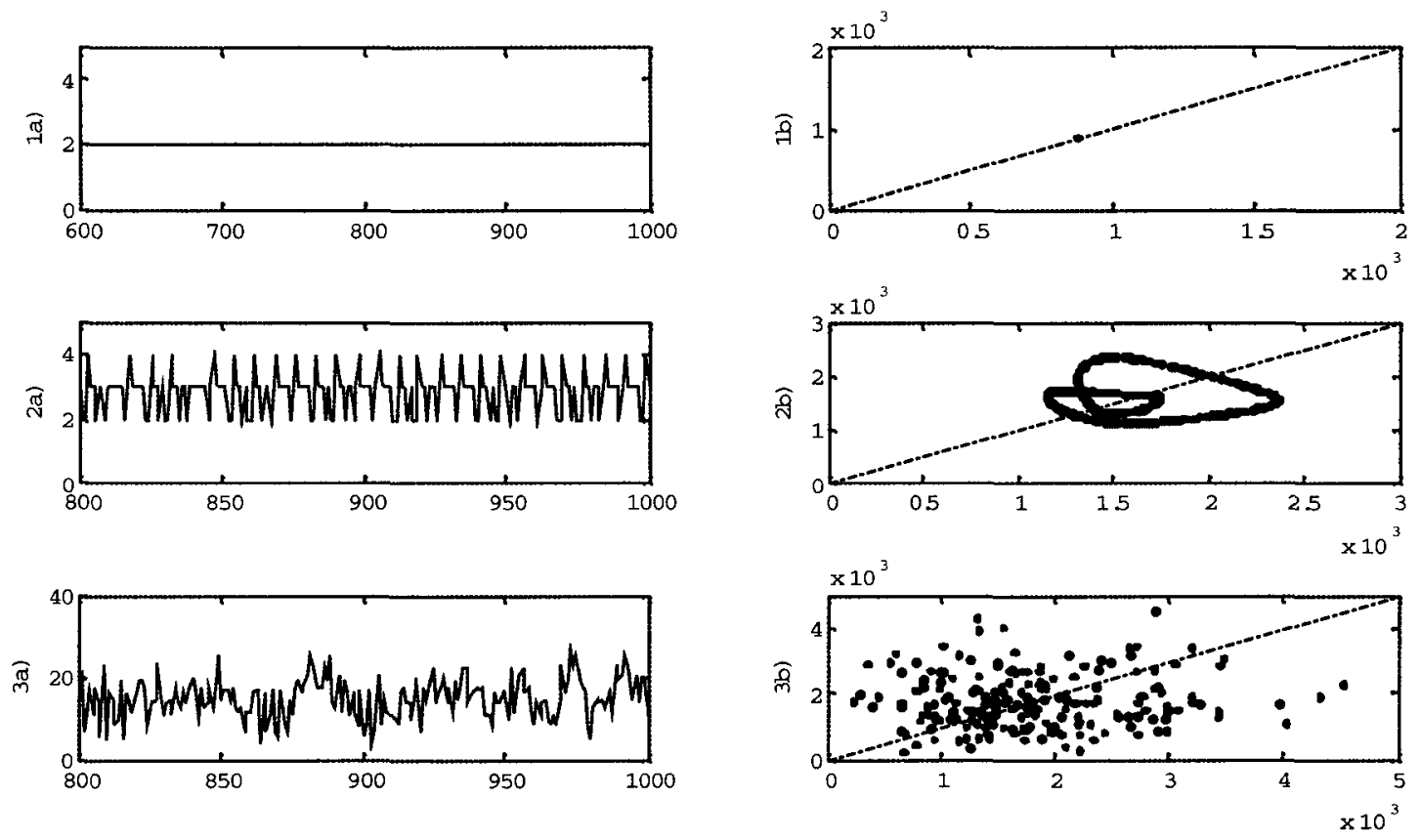

Figure 5.4: Iterative decoding trajectories for the $(1268,456) \mathrm{LDPC}$ code at $E_{b} / N_{0}=$ $2.5 \mathrm{~dB}$ : a) number of decision errors versus the iteration number. b) $\mathcal{E}(i+1)$ vs. $\mathcal{E}(i) ; 1)$ fixed-pattern 2 ) periodic-pattern, and 3) random-like or chaotic. 
Table 5.1: Breakdown of errors based on their type for the $(1268,456)$ code, decoded by standard $\mathrm{BP}$ and adaptive $\mathrm{BP}$, as a function of the maximum number of iterations at different SNR values; "C", "P" and "F" stand for "Chaotic", "Periodic" and "Fixed-pattern", respectively.

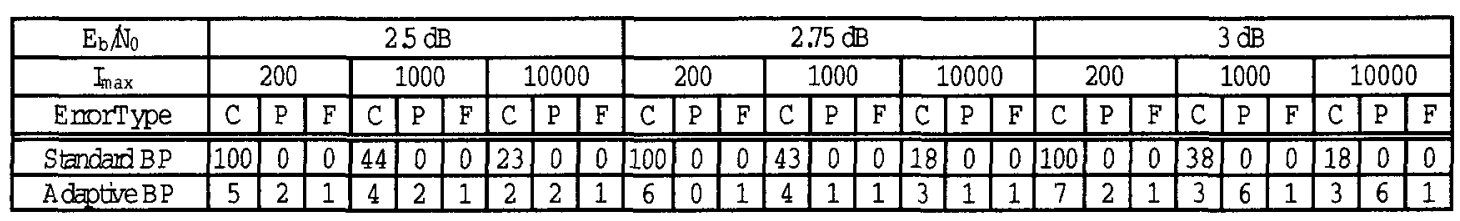

Table 5.2: Breakdown of errors based on their type for the $(504,252)$ code, decoded by standard $\mathrm{BP}$ and adaptive $\mathrm{BP}$, as a function of the maximum number of iterations at different SNR values; "C", "P" and "F" stand for "Chaotic", "Periodic" and "Fixed-pattern", respectively.

\begin{tabular}{|c|c|c|c|c|c|c|c|c|c|c|c|c|c|c|c|c|c|c|c|c|c|c|c|c|}
\hline$E_{b} \mathbb{N}_{0}$ & \multicolumn{8}{|c|}{$35 \mathrm{~dB}$} & \multicolumn{8}{|c|}{$4 \mathrm{~dB}$} & \multicolumn{8}{|c|}{$4.5 \mathrm{~dB}$} \\
\hline$I_{\max }$ & 20 & & & 1000 & & & $\overline{100}$ & & & 200 & & & $\overline{1000}$ & & 100 & & & 200 & & & $\overline{000}$ & & 100 & 00 \\
\hline EnorType & \begin{tabular}{|l|l|} 
C & P \\
\end{tabular} & $\bar{F}$ & 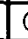 & P & $\mathrm{F}$ & $\mathrm{C}$ & $\mathrm{P}$ & $\bar{F}$ & $\bar{C}$ & P & $F$ & $\mathrm{C}$ & $P$ & $\mathrm{~F}$ & \begin{tabular}{l|l}
$\mathrm{C}$ & $\mathrm{P}$ \\
\end{tabular} & F & $\mathrm{C}$ & $\mathrm{P}$ & $F$ & c & $\overline{\mathrm{P}}$ & $\bar{F}$ & & IF \\
\hline Standard BP & \begin{tabular}{|l|l|}
100 & 0 \\
\end{tabular} & $\overline{0}$ & $\overline{4}$ & 0 & $\overline{0}$ & $\overline{15}$ & 0 & 0 & 100 & 0 & 0 & 29 & 1 & 0 & \begin{tabular}{l|l}
9 & 2 \\
\end{tabular} & $\overline{0}$ & 100 & 0 & 0 & 24 & $\overline{1}$ & $\overline{0}$ & 11 & 0 \\
\hline A daptive $B P$ & \begin{tabular}{|l|l|}
10 & 2 \\
\end{tabular} & $\overline{0}$ & & 2 & 0 & 2 & 2 & $\overline{0}$ & 6 & 5 & 4 & 5 & 5 & 4 & \begin{tabular}{l|l}
4 & 5 \\
\end{tabular} & 4 & 6 & 7 & 2 & 2 & 7 & & & 2 \\
\hline
\end{tabular}

respectively. As can be seen, for $I_{\max }=200$, all the 100 failure instances of standard BP for both codes and at all SNR values are chaotic. The results also show that as $I_{\max }$ increases to 1000 and then to 10,000 , many of these chaotic instances converge to the right codeword, indicating the transient nature of the chaos. For both codes, at each SNR, we apply the input vectors corresponding to the 100 failure instances of standard BP to adaptive BP. The breakdown of error types in this case is also reported in Tables 5.1 and 5.2. For example, Table 5.1 shows that for the $(1268,456)$ code, by applying the 100 input vectors, which caused chaos for the standard BP with $I_{\max }=200$ at SNR $=2.5 \mathrm{~dB}$, to the adaptive BP decoder with $I_{\max }=200$, only 5 cases remain in chaos. Out of the other 95 cases, 92 converge to the right codeword, 2 are trapped in periodic patterns and one in a fixed pattern. The results of Tables 5.1 and 5.2 indicate that the improvement in performance of adaptive BP in comparison 
with standard BP in the high $I_{\max }$ region is mainly due to the reduction in the chaotic transient behavior. This effect is particularly prevalent for smaller values of $I_{\max }$. 


\section{Chapter 6}

\section{Conclusions and Future Work}

In this thesis we have studied iterative decoding algorithms and methods to improve their performance. The main conclusions and proposed future work for each problem are summarized below.

- Belief Propogation algorithm due to the existence of cycles in the Tanner graph of practical codes is suboptimal. Improving the performance of BP is possible by normalizing the messages passed from variable nodes to check nodes in the iterative decoder. We have proposed an adaptive normalization scheme to improve the performance of BP algorithm.

- Inspired by the dynamics of SR, we propose a binary message-passing algorithm for the decoding of LDPC codes. Similar to SR, the proposed algorithm is with memory and the memory elements are updated differentially in each iteration according to the binary messages. The proposed algorithm is able to close a large portion of the gap between purely hard- decision and soft-decision algorithms.

- Successive Relaxation (SR) implementation of MS algorithm has shown to achieve better bit error rates in comparison to conventional Successive Substitution (SS) implementations of MS. In SR-MS algorithm, variable node massages are updated according to a different trajectory. As a result SR-MS generally 
requires more number of iterations to converge. A further study on behavior of orbits of SR-MS and devising a scheme to speed up the convergence while maintaining the performance seems promising.

- The proposed adaptive normalized BP algorithm improves the BER performance in expense of mild added complexity. The adaptive scaling factor $\alpha e^{-\beta \mid \mathbf{m}_{v \rightarrow c \mid}}$ was originally chosen in $[3,5,5]$ to speed up the convergence. But there might be other non-linear scaling functions that can perform better. A further search for discovering other alternative scaling functions makes an interesting research topic. 


\section{List of References}

[1] R. Gallager, "Low-density parity-check codes," IRE Trans. Inform. Theory, vol. 8, pp. 21-28, 1962.

[2] D. MacKay and R. Neal, "Near Shannon limit performance of low density parity check codes," Electronics Letters, vol. 33, pp. 457 - 458, Aug. 1997.

[3] D. MacKay, "Good error-correcting codes based on very sparse matrices," IEEE Trans. Inform. Theory, vol. 45, pp. 399 - 431, March 1999.

[4] IEEE P802.3an, "1OGBASE-T task force," http: //www. ieee8O2.org/3/an.

[5] IEEE 802.16e, "Air interface for fixed and mobile broadband wireless access systems," IEEE p802.16e/d12 draft, Feb. 2005.

[6] IEEE 802.11n, "Wireless lan medium access control and physical layer specifications," IEEE p802.11n/d3.07, March 2008.

[7] "T.T.S.I. digital video broadcasting (DVB) second generation framing structure for broadband satellite applications," http://www.dvb.org.

[8] M. Fossorier, M. Mihaljevic, and H. Imai, "Reduced complexity iterative decoding of low-density parity check codes based on belief propagation," IEEE Trans. Commun., vol. 47, pp. 673 -680, May 1999.

[9] J. Chen, A. Dholakia, E. Eleftheriou, M. Fossorier, and X.-Y. Hu, "Reducedcomplexity decoding of LDPC codes," IEEE Trans. Commun., vol. 53, pp. 1288 -1299 , July 2005.

[10] M. Fossorier, "Iterative reliability-based decoding of low-density parity check code," IEEE J. Select. Areas Commun., vol. 19, pp. 908 - 917, May 2001.

[11] M. Yazdani, S. Hemati, and A. Banihashemi, "Improving belief propagation on graphs with cycles," IEEE Comm. Letters, vol. 8, pp. 57 - 59, Jan. 2004. 
[12] Y. Mao and A. Banihashemi, "Decoding low-density parity-check codes with probabilistic scheduling," IEEE Commun. Letters, vol. 5, pp. $414-416$, Oct. 2001.

[13] S. Hemati and A. Banihashemi, "Dynamics and performance analysis of analog iterative decoding for low-density parity-check (LDPC) codes," IEEE Trans. on Commun., vol. 54, pp. 61 - 70, Jan. 2006.

[14] H. Xiao, S. Tolouei, and A. Banihashemi, "Successive relaxation for decoding of LDPC codes," in 24th Biennial Symposium on Communications, pp. $107-110$, June 2008.

[15] H. Xiao and A. H. Banihashemi, "Comments on successive relaxation for decoding of LDPC codes," IEEE Trans. on Comm., vol. 57, pp. 2846 - 2848, Oct. 2009.

[16] J. Proakis, Digital Communications. McGraw-Hill Series, 2000.

[17] S. Lin and D. Costello, Error Control Coding: Fundamentals And Applications. Prentice-Hall, Englewood Cliffs, NJ, 2004.

[18] C. Shannon, "A mathematical theory of communication," Bell System Technical Journal, vol. 27, pp. 379 - 423, 623 - 656, July and October 1948.

[19] R. Hamming, "Error detecting and error correcting codes," Bell Syst. Tech. J., vol. 29, pp. 147 - 160, April 1950.

[20] R. Bose and D. Ray-Chaudhuri, "On a class of error correcting binary group codes," Information and Control, vol. 3, pp. 68 - 79, March 1960.

[21] A. Hocquenghem, "Codes correcteurs derreurs," Chiffres, vol. 2, pp. 147 - 156, September 1959.

[22] I. Reed, , and G. Solomon, "Polynomial codes over certain finite fields," Journal of the Society for Industrial and Applied Mathematics, vol. 8, pp. 300 - 304, June 1960.

[23] P. Elias, "Coding for noisy channels," IRE Conv. Rec., vol. 4, pp. 37-46, Sept. 1955.

[24] A. Viterbi, "Error bounds for convolutional codes and an asymptotically optimum decoding algorithm," IEEE Trans. on Inform. Theory, vol. IT-13, pp. 260269, February 1967. 
[25] R. Gallager, Low-Density Party-Check Codes. PhD thesis, Cambridge, MA: MIT Press, 1963.

[26] R. Blahut, Theory and Practıce of Error Control Codes. Addison-Weseley Publishing Co., 1984.

[27] R. Tanner, "A recursive approach to low complexity codes," IEEE Trans. Inform. Theory, vol. 27, pp. $533-547$, Sept. 1981.

[28] M. Davey and D. MacKay, "Low-density parity check codes over GF(q)," IEEE Comm. Letters, vol. 2, pp. 165 - 167, June 1998.

[29] D. MacKay, "Gallager codes that are better than Turbo codes," in in Proc. 36th Allerton Conf. Communication, Control, and Computing, Montıcello, IL, Sept. 1998.

[30] Y. Kou, S. Lin, and M. Fossorier, "Low-density parity-check codes based on finite geometries: a rediscovery and new results," IEEE Trans. Inform. Theory, vol. 47 , pp. $2711-2736$, Nov. 2001.

[31] P. Zarrinkhat and A. Banihashemi, "Threshold values and convergence properties of majority-based algorithms for decoding regular low-density parity-check codes," IEEE Trans. Commun., vol. 52, pp. 2087 - 2097, Dec. 2004.

[32] T. Richardson and R. Urbanke, "The capacity of low-density parity-check codes under message-passing decoding," IEEE Trans. Inform. Theory, vol. 47, pp. 599 -618, Feb. 2001.

[33] H. Xiao and A. H. Banihashemi, "Graph-based message-passing schedules for decoding LDPC codes," IEEE Trans. Comm., vol. 52, pp. 2098 - 2105, Dec. 2004 .

[34] J. Zhang and M. Fossorier, "A modified weighted bit-flipping decoding of lowdensity parity-check codes," IEEE Commun. Letters, vol. 8, pp. 165 -167, March 2004 .

[35] M. Jiang, C. Zhao, Z. Shi, and Y. Chen, "An improvement on the modified weighted bit flipping decoding algorithm for LDPC codes," IEEE Communıcatrons Letters, vol. 9, pp. $814-816$, Sept. 2005.

[36] L. Kocarev, F. Lehmann, G. Maggio, B. Scanavino, Z. Tasev, and A. Vardy, "Nonlinear dynamics of iterative decoding systems: Analysis and applications," IEEE Trans. Inform. Theory, vol. 52, pp. 1366 - 1384, April 2006. 
[37] S. Hemati, Iteratıve Decoding in Analog VLSI. PhD thesis, Ottawa-Carleton Institute For Electrical and Computer Engineering, 2005.

[38] T. K. Moon and W. C. Stirling, Mathematical Methods and Algorithms for Signal Processing. Prentice Hall, 1999.

[39] Z. Liu and D. A. Pados, "A decoding algorithm for finite-geometry LDPC codes," IEEE Trans. Comm., vol. 53, pp. 415-421, April 2005.

[40] T. M. N. Ngatched, F. Takawira, and M. Bossert, "A modified bit-flipping decoding algorithm for low-density parity-check codes," in IEEE International Conference on Commun. ICC '07, pp. 653-658, August 2007.

[41] P. Moqvist and T. M. Aulin, "Turbo-decoding as a numerical analysis problem," in Information Theory, 2000. Proceedings. IEEE Internatıonal Symposium on, p. 485 , June 2000.

[42] E. Yeo, P. Pakzad, B. Nikolic, and V. Anantharam, "High throughput lowdensity parity-check decoder architectures," in IEEE Global Telecommunicatıons Conference, GLOBECOM '01, vol. 5, pp. 3019 - 3024, Nov. 2001.

[43] J. Dielissen, A. Hekstra, and V. Berg, "Low cost LDPC decoder for DVB-S2," in DATE Designers' Forum 2006, pp. 130 - 135, March 2006.

[44] J. Yedidia, W. Freeman, and Y. Weiss, "Constructing free-energy approximations and generalized belief propagation algorithms," IEEE Trans. on Inform. Theory, vol. 51 , pp. $2282-2312$, July 2005 .

[45] S. Hemati and A. Banihashemi, "On the dynamics of continuous-time analog iterative decoding," in IEEE International Symposıum on Information Theory, ISIT '04, p. 262, June 2004.

[46] A. Amat, S. Benedetto, G. Montorsi, D. Vogrig, A. Neviani, and A. Gerosa, "Design, simulation, and testing of a CMOS analog decoder for the block length40 UMTS Turbo code," IEEE Trans. Comm., vol. 54, pp. 1973 - 1982, Nov. 2006.

[47] Y. Mao and A. Banihashemi, "A heuristic search for good low-density paritycheck codes at short block lengths," in IEEE International Conference on Communıcations, ICC 2001, vol. 1, pp. 41 - 44, June 2001.

[48] D. J. C. MacKay, "Encyclopedia of sparse graph codes [online]," in Avallable at: http://www. inference.phy.cam.ac.uk/mackay/codes/data.html. 
[49] N. Mobini, A. Banihashemi, and S. Hemati, "A differential binary message passing LDPC decoder," in IEEE Global Telecommunıcatıons Conference, GLOBECOM '07, pp. 1561 - 1565, Nov. 2007.

[50] N. Mobini, A. Banihashemi, and S. Hemati, "A differential binary messagepassing ldpc decoder," IEEE Trans. on Commun., vol. 57, pp. 2518 - 2523, Sept. 2009.

[51] A. J. Blanksby and C. J. Howland, "A 690-mW 1-Gb/s 1024-b, rate-1/2 lowdensity parity-check code decoder," IEEE J. Solıd-State Circunts, vol. 37, pp. 404 -412 , March 2002.

[52] S. T. Brink, G. Kramer, and A. Ashikhmin, "Design of low-density parity check codes for modulation and detection," IEEE Trans. Comm., vol. 52, pp. 670 678, April 2004.

[53] E. Janulewicz and A. Banihashemi, "Performance analysis of iterative decoding algorithms with memory," in IEEE Informatıon Theory Workshop (ITW), pp. 1 - 5, Jan. 2010.

[54] H. Xiao and A. Banihashemi, "Improved progressive-edge-growth (PEG) construction of irregular LDPC codes," IEEE Comm. Letters, vol. 8, pp. 715 - 717, Dec. 2004.

[55] C. Berrou, A. Glavieux, and P. Thitimajshima, "Near shannon limit errorcorrecting coding and decoding: Turbo-codes," in Technıcal program, conference record, IEEE international conference on Communications, ICC 93, vol. 2, pp. $1064-1070$, May 1993.

[56] D. MacKay and M. Davey, "Evaluation of gallager codes for short block length and high rate applications," Codes, Systems and Graphical Models, vol. 123, pp. 113-130, 1999.

[57] R. Lucas, M. Fossorier, Y. Kou, and S. Lin, "Iterative decoding of one-step majority logic deductible codes based on belief propagation," Communıcatıons, IEEE Transactions on, vol. 48, pp. 931-937, June 2000.

[58] L. Kocarev, Z. Tasev, and A. Vardy, "Improving turbo codes by control of transient chaos in turbo-decoding algorithms," Electronıcs Letters, vol. 38, pp. 1184 - 1186, Sep. 2002. 